\title{
Turbulent diffusion and mixing in interstellar dark clouds with adsorption onto grains
}

\author{
C. J. Yate and T. J. Millar
}

\begin{abstract}
Department of Physics, UMIST, PO Box 88, Manchester M60 1QD, UK
Received 17 April 2002 / Accepted 6 December 2002

Abstract. This paper reports the results of models of dark cloud chemistry incorporating a depth dependent density distribution with diffusive mixing and adsorption onto grains. The model is based on the approach taken by Xie et al. (1995), with the addition of grain accretion effects. Without diffusion, the central regions of the cloud freeze out in less than $10^{7}$ years. Freezeout time is dependent on density, so the diffuse outer region of the cloud remains abundant in gas for about an order of magnitude longer. We find that fairly small amounts of diffusive mixing can delay freeze-out at the centre of the model cloud for a time up to an order of magnitude greater than without diffusion, due to material diffusing inward from the edges of the cloud. The gas-phase lifetime of the cloud core can thus be increased by up to an order of magnitude or more by this process. We have run three different grain models with various diffusion coefficients to investigate the effects of changing the sticking parameters.
\end{abstract}

Key words. astrochemistry - turbulence - diffusion - ISM: abundances - ISM: dust, extinction

\section{Introduction}

The evidence for turbulence within interstellar clouds comes from widths of molecular emission and absorption lines which can be broader than would be possible from thermal broadening alone. Turbulence occurs in fluid flows where the Reynolds number exceeds the range 100 to 10000 . Scalo (1987) calculated a Reynolds number $R e \sim 10^{8} l_{\mathrm{pc}}^{-0.7}$ for interstellar gas, where $l_{\mathrm{pc}}$ is the scale length of the cloud in parsecs. For a typical dark cloud of radius $1 \mathrm{pc}$ this gives $R e \sim 10^{8}$ - clearly suggesting material in dark cloud cores of this scale should be turbulent.

Line broadening in spectroscopic observations of interstellar gas can be caused by thermal or collisional broadening, or macroscopic random motion due to turbulence. Collisional broadening is only important at very high densities and high temperature, so will not be important in the cold, dark clouds considered in this work. A considerable fraction of line broadening in clouds is due to turbulent motion. Larson (1981) and Leung et al. (1982, hereafter LKM) studied linewidths in clouds and found a relationship between cloud size and line broadening due to turbulence. This is discussed in Sect. 4 below.

Turbulence within a cloud causes mixing of the gas. This can affect the chemistry as reactants are redistributed throughout the cloud. Turbulence is extremely difficult to model accurately as there is, as yet, no rigorous mathematical description of the processes involved. However there have been various attempts to model mixing of the gas in clouds, e.g. the models of Chièze \& Pineau des Forêts (1989), Chièze et al. (1991),

Send offprint requests to: T. J. Millar, e-mail: Tom.Millar@umist.ac.uk
Pineau des Forêts et al. (1992), Xie et al. (1995), Rawlings \& Hartquist (1997), and Willacy et al. (2002).

Chièze \& Pineau des Forêts (1989) developed models where two regions, one dense and one diffuse, exchanged packets of gas phase material. Xie et al. (1995) extended this idea to model a one dimensional grid of points between the centre of a dark cloud core and the diffuse material surrounding it. Xie et al.'s models approximate diffusive mixing by calculating abundance gradients across the cloud. Diffusive mixing tends to smooth abundance gradients across the cloud. Willacy et al. (2002) have recently reported models based on Xie et al., with some added grain chemistry. Rawlings \& Hartquist (1997) considered the problem of diffusion across a thin boundary layer.

Xie et al. required relatively high levels of the diffusion coefficient, $K$ (see Eq. (3)), to significantly change their calculated abundances. In this work we have extended their work by including adsorption of material onto dust grains. This process is density dependent, resulting in large gradients across the cloud. As we shall see, diffusion can significantly alter chemical abundances. The larger gradients provided by grain accretion mean that significant changes to the chemical abundances occur for much smaller diffusion rates.

\section{Model details}

\subsection{Physical model and chemistry}

We neglect spherical coordinates for simplicity, and model the 1-D density profile into a slab of gas of thickness $1.1 \mathrm{pc}$. We divide the cloud into cells with boundaries given in Table 1, and evaluate the chemical abundances at the centre of each cell. The cell at $3.5 \times 10^{18} \mathrm{~cm}$ is assumed to be the edge of the cloud. These physical conditions are the same as used by 
Table 1. Physical conditions of the model.

\begin{tabular}{lrr}
\hline \hline Grid boundaries $(\mathrm{cm})$ & $A_{V}(\mathrm{mag})$ & Density $n_{\mathrm{H}_{2}}\left(\mathrm{~cm}^{-3}\right)$ \\
\hline $7.50 \times 10^{16}$ & 10.00 & $3.50 \times 10^{4}$ \\
$1.00 \times 10^{17}$ & 9.00 & $3.00 \times 10^{4}$ \\
$1.50 \times 10^{17}$ & 8.00 & $2.00 \times 10^{4}$ \\
$2.25 \times 10^{17}$ & 7.00 & $1.33 \times 10^{4}$ \\
$3.38 \times 10^{17}$ & 6.00 & $8.89 \times 10^{3}$ \\
$5.06 \times 10^{17}$ & 5.00 & $5.92 \times 10^{3}$ \\
$7.59 \times 10^{17}$ & 4.00 & $3.95 \times 10^{3}$ \\
$1.14 \times 10^{18}$ & 3.00 & $2.63 \times 10^{3}$ \\
$1.71 \times 10^{18}$ & 2.00 & $1.76 \times 10^{3}$ \\
$1.90 \times 10^{18}$ & 1.70 & $1.58 \times 10^{3}$ \\
$2.11 \times 10^{18}$ & 1.40 & $1.42 \times 10^{3}$ \\
$2.34 \times 10^{18}$ & 1.10 & $1.28 \times 10^{3}$ \\
$2.51 \times 10^{18}$ & 0.90 & $1.19 \times 10^{3}$ \\
$2.69 \times 10^{18}$ & 0.70 & $1.12 \times 10^{3}$ \\
$2.78 \times 10^{18}$ & 0.60 & $1.08 \times 10^{3}$ \\
$2.88 \times 10^{18}$ & 0.50 & $1.04 \times 10^{3}$ \\
$2.98 \times 10^{18}$ & 0.40 & $1.01 \times 10^{3}$ \\
$3.08 \times 10^{18}$ & 0.30 & $9.74 \times 10^{2}$ \\
$3.19 \times 10^{18}$ & 0.20 & $9.41 \times 10^{2}$ \\
$3.30 \times 10^{18}$ & 0.10 & $9.10 \times 10^{2}$ \\
$3.33 \times 10^{18}$ & 0.07 & $9.00 \times 10^{2}$ \\
$3.38 \times 10^{18}$ & 0.03 & $8.89 \times 10^{2}$ \\
$3.41 \times 10^{18}$ & 0.00 & $8.86 \times 10^{2}$ \\
$3.50 \times 10^{18}$ & 0.00 & $8.50 \times 10^{2}$ \\
\hline & &
\end{tabular}

Xie et al. The cloud is assumed to be isothermal, with a constant temperature of $10 \mathrm{~K}$.

The chemistry is modelled by a network of 127 species with 1669 reactions. The species set includes molecules containing $\mathrm{H}, \mathrm{He}, \mathrm{C}, \mathrm{N}$ and $\mathrm{O}$, with $\mathrm{Na}$ included as a representative metal. Molecules containing up to 2 carbon atoms are included. Initial elemental fractional abundances taken from Leung et al. (1984) are shown in Table 2. The ordinary differential equations (ODEs) are solved by a Gear algorithm, using the CVODE solver (Hindmarsh \& Cohen 1994). The chemistry reaction rate coefficients are a subset of the UMIST ratefile (Le Teuff et al. 2000). $\mathrm{H}_{2}$ and $\mathrm{e}^{-}$abundances are calculated by means of algebraic conservation equations, with respect to sources and sinks of $\mathrm{H}$ and the sum of positive ions respectively. No negative ions apart from $\mathrm{e}^{-}$are included.

The cloud is divided into 23 cells, and the ODEs for all cells are solved together, so we integrate $23 \times 125$ ODEs simultaneously. This ensures that each cell is at the same time within the solver, and is particularly important when including the diffusion terms because the solver adjusts the time step depending on the stiffness of the problem at a particular time. If the chemistry is calculated separately for each cell, the diffusion calculation fails, as parts of the cloud can be at different times within the solver.

The ODEs are expressed in the form:

$\frac{\mathrm{d} n_{i}}{\mathrm{~d} t}=F_{i}-D_{i}-\frac{\mathrm{d} \phi_{i}}{\mathrm{~d} z}-A_{i}$

where for a species $i, n_{i}$ is the species density (fractional abundance multiplied by the total gas density $\left.n_{\mathrm{H}}\right), F_{i}$ and $D_{i}$ are
Table 2. Initial abundances.

\begin{tabular}{ll}
\hline \hline Species & $n_{i} / n_{\text {total }}$ \\
\hline $\mathrm{H}_{2}$ & 0.5 \\
$\mathrm{He}$ & 0.14 \\
$\mathrm{C}^{+}$ & $7.3 \times 10^{-5}$ \\
$\mathrm{~N}$ & $2.14 \times 10^{-5}$ \\
$\mathrm{O}$ & $1.76 \times 10^{-4}$ \\
$\mathrm{Na}^{+}$ & $1.9 \times 10^{-8}$ \\
\hline
\end{tabular}

total formation and destruction rates (per unit volume per second) due to chemistry, $\mathrm{d} \phi_{i} / \mathrm{d} z$ is the change per unit volume per second due to turbulent diffusion and $A_{i}$ is the loss rate of gas phase molecules onto grains, per unit volume per second.

\subsection{Turbulent diffusion calculation}

The diffusion calculation follows the approach described by Xie et al. (1995). The net flux per species $i$ at each grid point is:

$\phi_{i}=-K n\left(\mathrm{H}_{2}\right) \frac{\mathrm{d} f_{i}}{\mathrm{~d} z} \mathrm{~cm}^{-2} \mathrm{~s}^{-1}$

where $f_{i}$ is the fractional abundance of each species, $n\left(\mathrm{H}_{2}\right)$ is the density of molecular hydrogen, $z$ is the depth coordinate, and $K$ is the diffusion coefficient which sets the speed of diffusion, defined as:

$K=\left\langle v_{\mathrm{t}} L>\mathrm{cm}^{2} \mathrm{~s}^{-1}\right.$

where $v_{\mathrm{t}}$ is the turbulent velocity, measured from the linewidths of molecular lines (e.g. Larson 1981; LKM82; Myers 1983), and $L$ is the mixing length or scale length of the turbulence.

The turbulent mixing length within interstellar clouds is not well understood and is difficult to measure. The Kolmogorov microscale $l_{\mathrm{k}}$ where viscous dissipation of turbulence becomes important, is likely to be very small compared to the size scale of interstellar clouds. Scalo (1987) gives the formula $l_{\mathrm{k}}=$ $10^{12} l_{\mathrm{pc}}^{-1} \mathrm{~cm}$. So, for a cloud of radius $\sim 1 \mathrm{pc}$ we could reasonably expect turbulence to affect gas on scales down to $10^{12} \mathrm{~cm}$ $\left(\sim 10^{-6} \mathrm{pc}\right)$, as large eddies break down into smaller motions. Molecular abundance variations on this size scale are not observable with current instruments. However, an estimate of the size scale of the large eddies within a cloud can reveal some detail of the mixing processes involved.

Xie et al. used an order of magnitude estimate to obtain the value, $L=0.1-0.5 \mathrm{pc}$, based on the autocorrelation analysis of ${ }^{13} \mathrm{CO}$ in TMC-1, by Kleiner \& Dickman (1987, hereafter KD). Larson and LKM suggest $v_{\mathrm{t}}$ for a 1 pc cloud should be not much larger than $1 \mathrm{~km} \mathrm{~s}^{-1}$. A scale length for turbulence larger than $10 \%$ of the cloud size also seems unlikely - giving a maximum value for clouds this size of $K \simeq 10^{22.5} \mathrm{~cm}^{2} \mathrm{~s}^{-1}$. Therefore we use a maximum value of $K=10^{23} \mathrm{~cm}^{2} \mathrm{~s}^{-1}$. Larger values of $K$ quickly become prohibitively expensive in computing time. In this paper, we show results for $K$ of values of 0 , and between $10^{21}$ and $10^{23} \mathrm{~cm}^{2} \mathrm{~s}^{-1}$, to investigate the effects of different diffusion rates.

Initial abundance gradients at early model times are very large. As a result, calculating the turbulent mixing from $t=0$ 
causes numerical problems. To avoid this we introduce diffusion from 1000 years. This allows the chemistry to begin to develop, and is a short time in the age of the model cloud.

Following a suggestion by Rawlings \& Hartquist (1997), the maximum step size within the solver is set to be less than the smallest diffusion time. Rawlings \& Hartquist suggest a maximum step of $1 / 5$ of the shortest diffusion time. We have set the solver to a maximum step size $2 \times 10^{3}$ years, which should be sufficient. If material is allowed to move across a cell on a smaller timescale than that of the solver step size, the accuracy is affected and errors can occur.

\subsection{Grain processes}

Dust grains are ubiquitous in dense interstellar clouds. When most molecules collide with cold dust grains, it is very likely that the molecule will stick, forming a physical or chemical bond with the grain surface. Not all molecules stick to dust particles, and certain species are more likely to remain attached to grains than others. In a cloud at $10 \mathrm{~K}$ with no internal heat source, it is difficult for most molecules to escape the grains by thermal effects. Cosmic ray particles can cause some desorption even at the centre of the cloud, but this is a very slow effect and is neglected.

The rate coefficient for adsorption, $R_{i} \mathrm{~cm}^{3} \mathrm{~s}^{-1}$ of species $i$ onto a grain (d'Hendecourt et al. 1985) is:

$R_{i}=S_{i} \sigma_{\mathrm{g}} \overline{v_{i}} \mathrm{~cm}^{3} \mathrm{~s}^{-1}$,

where $S_{i}$ is the sticking probability for species $i, \sigma_{\mathrm{g}} \mathrm{cm}^{2}$ is the cross section of the grain and $\overline{v_{i}} \mathrm{~cm} \mathrm{~s}^{-1}$ is the mean thermal velocity of $i, \overline{v_{i}}=\left(8 k_{\mathrm{b}} T / \pi m_{i}\right)^{1 / 2} \mathrm{~cm} \mathrm{~s}^{-1}$, where $k_{\mathrm{b}}$ is Boltzmann's constant, and $m_{i}$ is the mass in grams of the species. The dust cross-section $\sigma_{\mathrm{g}}$ is written $\pi a_{\mathrm{g}}^{2} \mathrm{~cm}^{2}$ where $a_{\mathrm{g}}$ is the grain radius, assumed to be $10^{-5} \mathrm{~cm}$. This leads to the rate of adsorption onto the dust:

$A_{i}=R_{i} n_{\mathrm{g}} n_{i}$,

where $n_{\mathrm{g}}$ is the dust abundance assumed to be $10^{-12} \times$ $n\left(\mathrm{H}_{2}\right) \mathrm{cm}^{-3}$, and $n_{i}$ is the abundance of species $i \mathrm{~cm}^{-3}$.

We consider a number of different models with regard to sticking efficiencies. Our "normal" model, model A, follows typical parameters adopted by other workers, that is: $\mathrm{H}$ has a sticking coefficient $S_{\mathrm{H}}=1 / 3$, the species $\mathrm{H}^{+}, \mathrm{H}_{2}, \mathrm{H}_{2}^{+}, \mathrm{H}_{3}^{+}, \mathrm{He}$, $\mathrm{He}^{+}, \mathrm{HeH}^{+}$and $\mathrm{e}^{-}$all have sticking coefficients $S_{i}=0$, and sticking coefficients for all heavier species are $S_{i}=1$.

Model B investigates a lower sticking coefficient, $S_{i}=1 / 3$ for all heavier species.

Model C allows chemical reactions between the incoming positive ions and negatively charged grains. We include the reactions

$\mathrm{X}^{+}+\mathrm{gr}^{-} \rightarrow$ products

where the products return to the gas phase, and the adopted branching ratios follow those of the gas phase recombination reactions

$\mathrm{X}^{+}+\mathrm{e}^{-} \rightarrow$ products
The e-folding timescale for adsorption $t_{\mathrm{ads}}$, is given, with $T=$ $10 \mathrm{~K}$, by the formula:

$t_{\mathrm{ads}}=\left(1.44 \times 10^{-17} S_{i} \frac{n\left(\mathrm{H}_{2}\right)}{\sqrt{m_{i}}}\right)^{-1} \mathrm{~s}$.

The only variables in this equation are $S_{i}, n\left(\mathrm{H}_{2}\right)$ and $m_{i}$, where $m_{i}$ is the relative molecular mass. The mass term is to the half power, so is a factor of $<10$ for all the species in this model.

For example, at grid point $2, n\left(\mathrm{H}_{2}\right)=3 \times 10^{4} \mathrm{~cm}^{-3}$. Assuming a species with mass 20 and a sticking probability of unity, gives:

$t_{\text {ads }}=1.04 \times 10^{13} \mathrm{~s} \simeq 3.3 \times 10^{5} \mathrm{yr}$.

At the centre of the cloud, most species should be significantly frozen out after about $10^{6}$ years. At the outer edges the freezeout occurs at around $10^{7-8}$ years or greater. The large depth dependence on abundance which results can drive the diffusion faster once freeze-out starts to become dominant, generally at $t>10^{5.5}$ years.

\section{Results}

\section{1. $S=1.0-$ model $A$}

We first examine results for grain model $\mathrm{A}$, with varying diffusion coefficients. Two species, $\mathrm{CH}_{3} \mathrm{OH}$ and $\mathrm{C}_{2} \mathrm{H}$ are discussed, with $K=0, K=10^{21}, K=10^{22}, K=10^{23} \mathrm{~cm}^{2} \mathrm{~s}^{-1}$. Also plotted are $\mathrm{H}, \mathrm{H}_{2} \mathrm{O}, \mathrm{H}_{2} \mathrm{CO}, \mathrm{NH}_{3}, \mathrm{CN}, \mathrm{HCN}$ and $\mathrm{HCO}^{+}$.

\subsubsection{General effects on species abundance}

Figures 1 to 9 show the effects of varying the diffusion constant on the fractional abundances of $\mathrm{CH}_{3} \mathrm{OH}, \mathrm{C}_{2} \mathrm{H}, \mathrm{NH}_{3}, \mathrm{H}_{2} \mathrm{O}$, $\mathrm{H}_{2} \mathrm{CO}, \mathrm{CN}, \mathrm{HCN}, \mathrm{HCO}^{+}$and $\mathrm{H}$ respectively, with four values of $K: 0,10^{21}, 10^{22}$ and $10^{23} \mathrm{~cm}^{2} \mathrm{~s}^{-1}$. All of these species, with the exception of $\mathrm{H}$ show the same global behaviour. For $K=0 \mathrm{~cm}^{2} \mathrm{~s}^{-1}$, the species are completely removed at the cloud centre on a time-scale of $\sim 10^{6} \mathrm{yrs}$, while at the edge the timescale is $\sim 10^{7.6}$ yrs. Increasing $K$ has the effect of increasing the adsorption time at the centre and decreasing it at the edge, making the adsorption timescale around $10^{7} \mathrm{yrs}$ in both regions. We shall discuss the particular cases of $\mathrm{CH}_{3} \mathrm{OH}$, which shows a large centre to edge abundance gradient for the case $K=0 \mathrm{~cm}^{2} \mathrm{~s}^{-1} ; \mathrm{C}_{2} \mathrm{H}$, which has a fairly shallow centre to edge gradient; and $\mathrm{H}$ atoms, which Willacy et al. (2002) have argued are sensitive to $K$.

Figure 1 shows the fractional abundance of $\mathrm{CH}_{3} \mathrm{OH}$ as a function of time, at various grid points.

With $K=0 \mathrm{~cm}^{2} \mathrm{~s}^{-1}, \mathrm{CH}_{3} \mathrm{OH}$ reaches a maximum fractional abundance $\sim 2 \times 10^{-10}$ at the central grid point, at $10^{5.2}$ years. From this time onward, the abundance decreases, as $\mathrm{CH}_{3} \mathrm{OH}$ formation slows and molecules freeze onto grains. At the centre, $\mathrm{CH}_{3} \mathrm{OH}$ abundance drops below $10^{-20}$ at $10^{6.3}$ years. Many other molecules freeze out at or around this time, which is dependent on both the mass of the molecule and the particular chemical reactions which affect 
$\mathrm{CH} 3 \mathrm{OH}$ abundance $\mathrm{S}=1 \mathrm{k}=0$

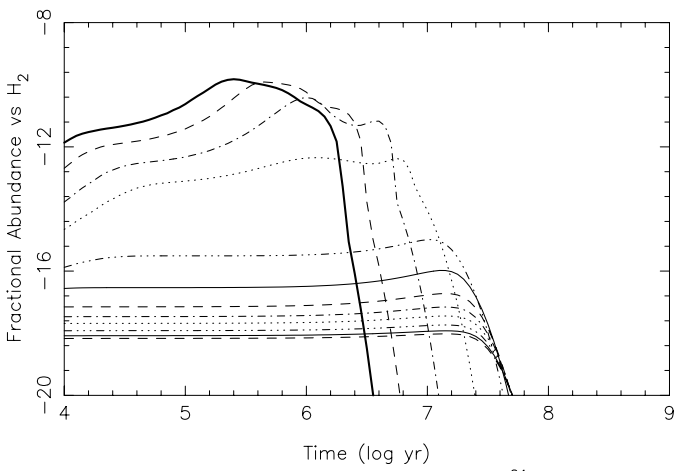

$\mathrm{CH} 3 \mathrm{OH}$ abundance $\mathrm{S}=1 \mathrm{k}=10^{21}$

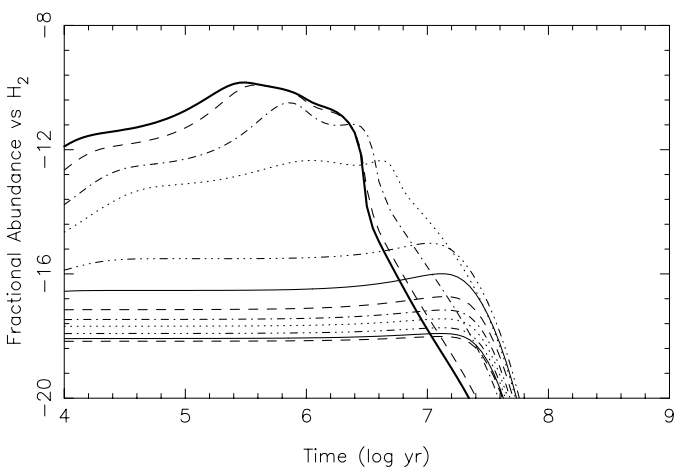

$\mathrm{CH} 3 \mathrm{OH}$ abundance $\mathrm{S}=1 \mathrm{k}=10^{22}$

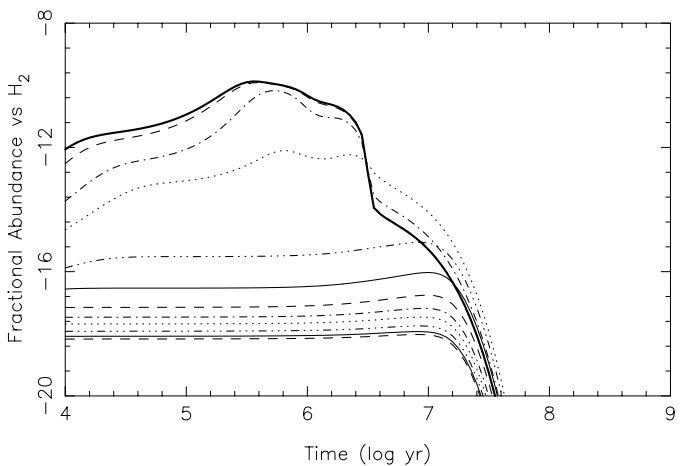

$\mathrm{CH} 3 \mathrm{OH}$ abundance $\mathrm{S}=1 \mathrm{k}=10^{23}$

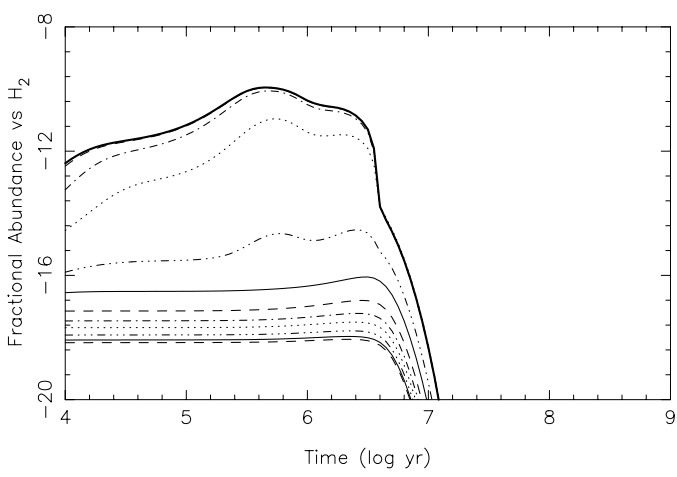

Fig. 1. $\mathrm{CH}_{3} \mathrm{OH}$ abundances, grain model A. A heavy solid line marks the centre of cloud, each line plotted shows each alternate gridpoint.

the species. With a diffusion coefficient $K=10^{21} \mathrm{~cm}^{2} \mathrm{~s}^{-1}$, the abundance of $\mathrm{CH}_{3} \mathrm{OH}$ at the centre of the cloud is about 5 orders of magnitude larger at $10^{6.2}$ years than the diffusionless case. The fractional abundance at the centre is also maintained above $10^{-20}$ for over $10^{7}$ years, considerably longer
C2H abundance $\mathrm{S}=1 \mathrm{k}=0$

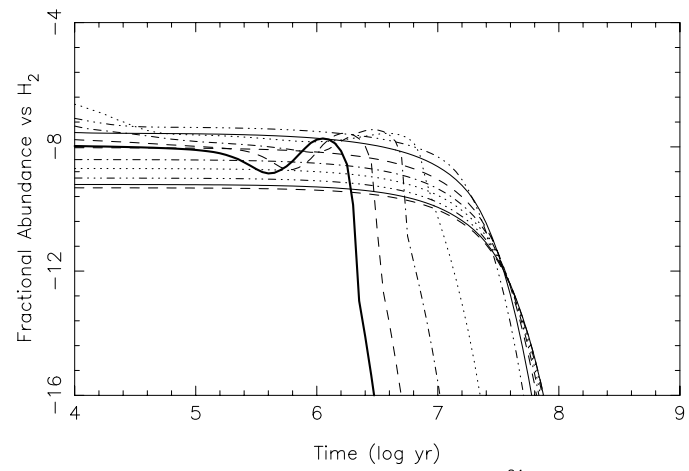

$\mathrm{C} 2 \mathrm{H}$ abundance $\mathrm{S}=1 \mathrm{k}=1 \mathrm{O}^{2}$

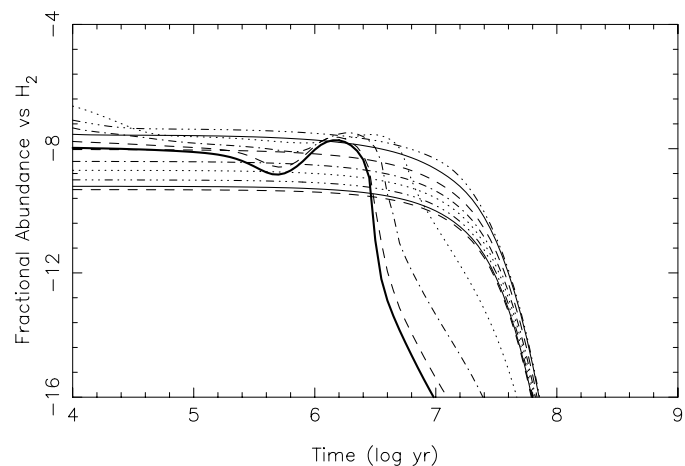

$\mathrm{C} 2 \mathrm{H}$ abundance $\mathrm{S}=1 \mathrm{k}=1 \mathrm{O}^{22}$

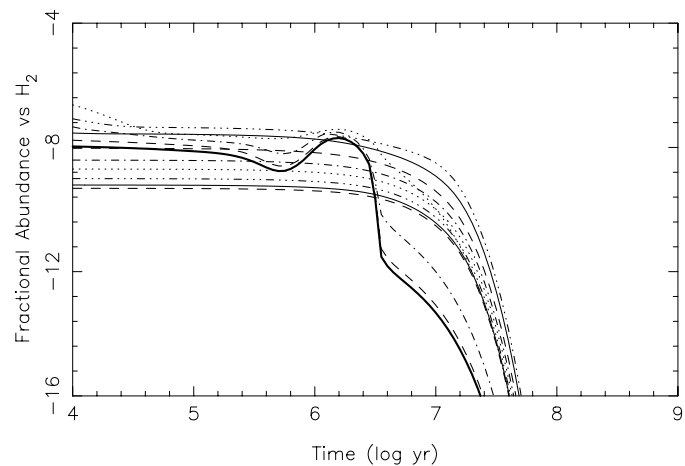

$\mathrm{C} 2 \mathrm{H}$ abundance $\mathrm{S}=1 \mathrm{k}=1 \mathrm{O}^{23}$

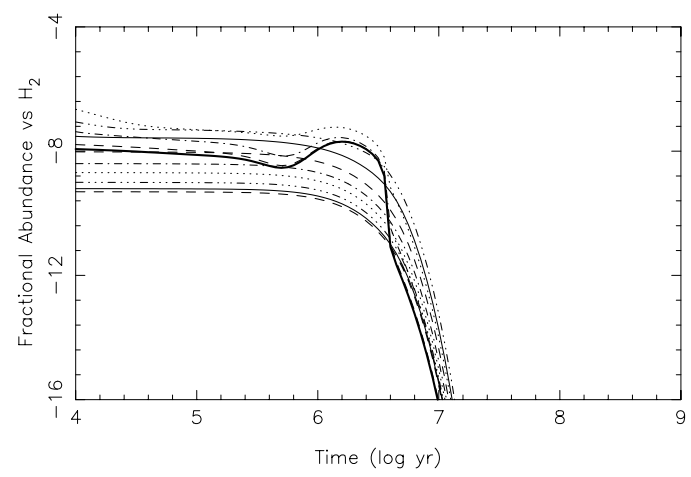

Fig. 2. $\mathrm{C}_{2} \mathrm{H}$ abundances. Grain model $\mathrm{A}$.

than in the $K=0 \mathrm{~cm}^{2} \mathrm{~s}^{-1}$ model. For the $K=10^{22} \mathrm{~cm}^{2} \mathrm{~s}^{-1}$ model, $\mathrm{CH}_{3} \mathrm{OH}$ is around 2 orders of magnitude more abundant at $10^{7}$ years, at the centre compared to the abundance with $K=10^{21} \mathrm{~cm}^{2} \mathrm{~s}^{-1}$. With $K=10^{23}$, the $\mathrm{CH}_{3} \mathrm{OH}$ abundance at the centre drops more sharply, and in contrast to the models with smaller diffusion coefficients, its abundance is greater at 
NH3 abundance $S=1 k=0$

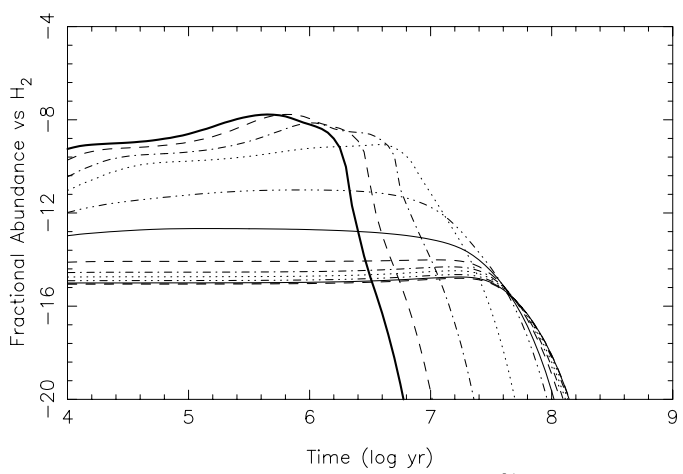

$\mathrm{NH} 3$ abundance $\mathrm{S}=1 \mathrm{k}=10^{21}$

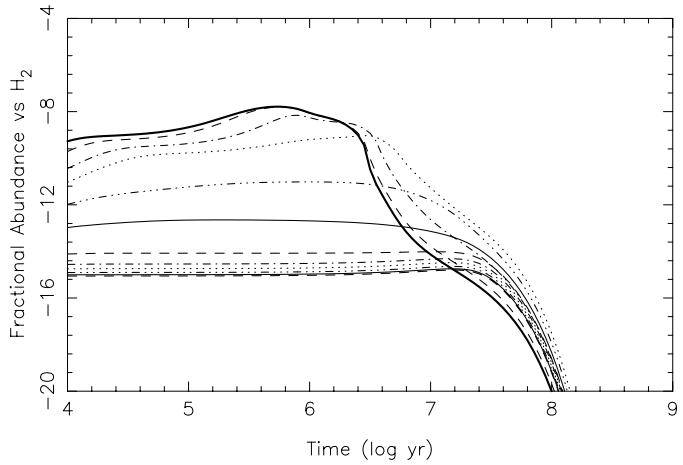

$\mathrm{NH} 3$ abundance $\mathrm{S}=1 \mathrm{k}=10^{22}$

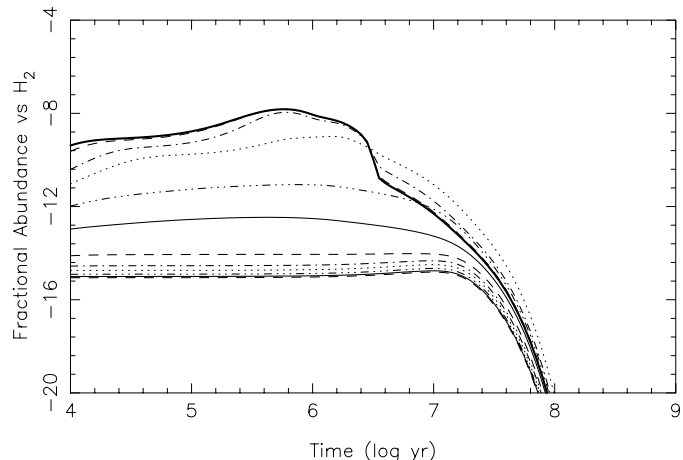

NH3 abundance $S=1 \quad k=10^{23}$

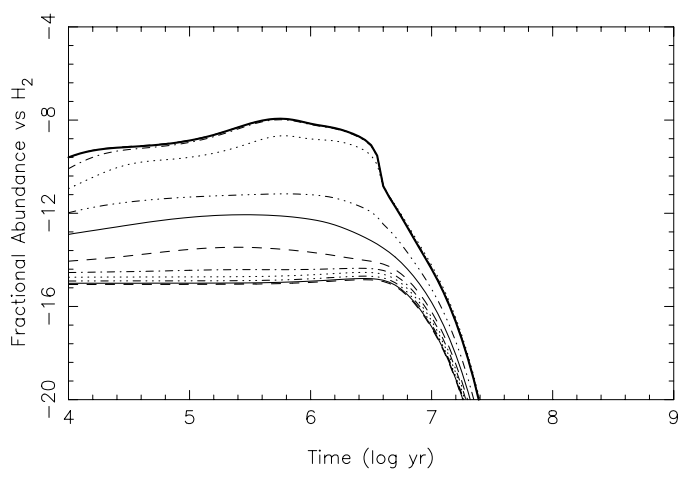

Fig. 3. $\mathrm{NH}_{3}$ abundances. Grain model A.

the centre than at any other point in the cloud, from $10^{4}$ years onward. The fractional abundance also decreases faster at the edge of the cloud. $\mathrm{CH}_{3} \mathrm{OH}$ crosses the time axis with a fractional abundance $10^{-20}$, at $10^{6.8}$ years for the edge shell, compared to $10^{7.7}$ years in the $K=0 \mathrm{~cm}^{2} \mathrm{~s}^{-1}$ model. At the centre of the cloud the $\mathrm{CH}_{3} \mathrm{OH}$ abundance follows a similar trend to
H2O abundance $S=1 \quad k=0$

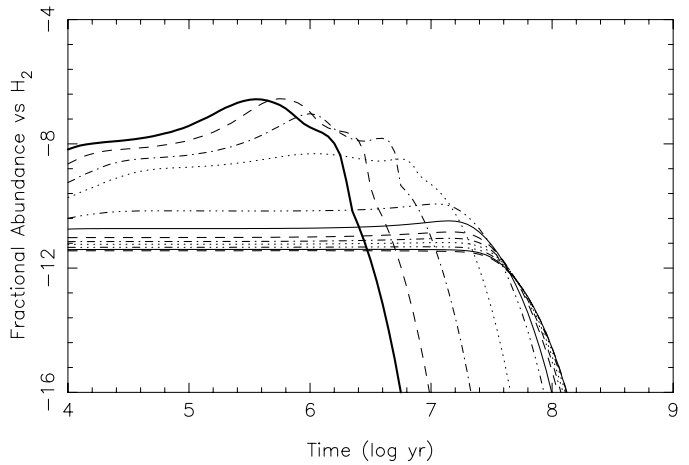

$\mathrm{H} 2 \mathrm{O}$ abundance $\mathrm{S}=1 \mathrm{k}=1 \mathrm{O}^{2}$

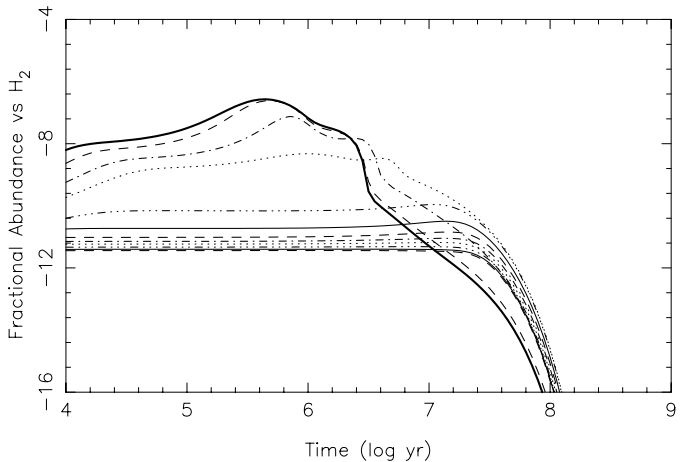

H2O abundance $S=1 \quad k=10^{22}$

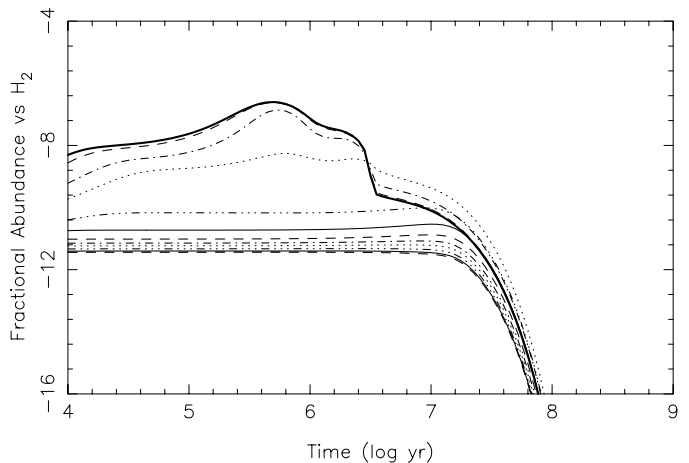

H2O abundance $S=1 \quad k=10^{23}$

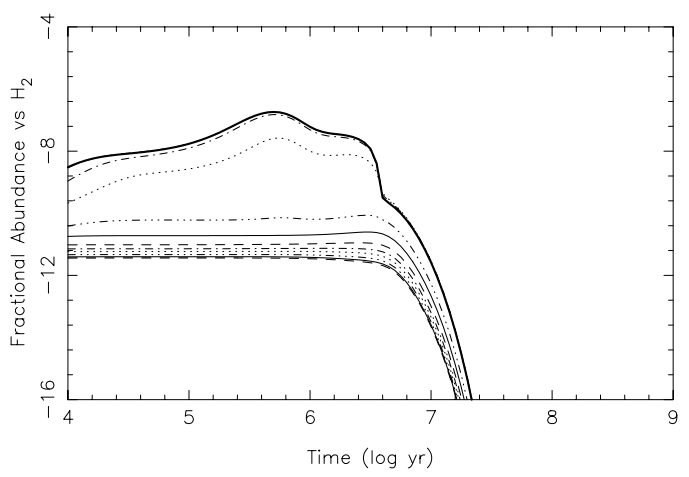

Fig. 4. $\mathrm{H}_{2} \mathrm{O}$ abundances. Grain model A.

that seen in the $K=10^{22} \mathrm{~cm}^{2} \mathrm{~s}^{-1}$ plot, but drops off much more sharply.

At early times, before freeze-out dominates, $\mathrm{CH}_{3} \mathrm{OH}$ is found to have higher abundances in the centre of a cloud, where the molecules are shielded from interstellar UV radiation. This is very clear in the models, as there is a difference 
$\mathrm{H} 2 \mathrm{CO}$ abundance $\mathrm{S}=1 \mathrm{k}=0$

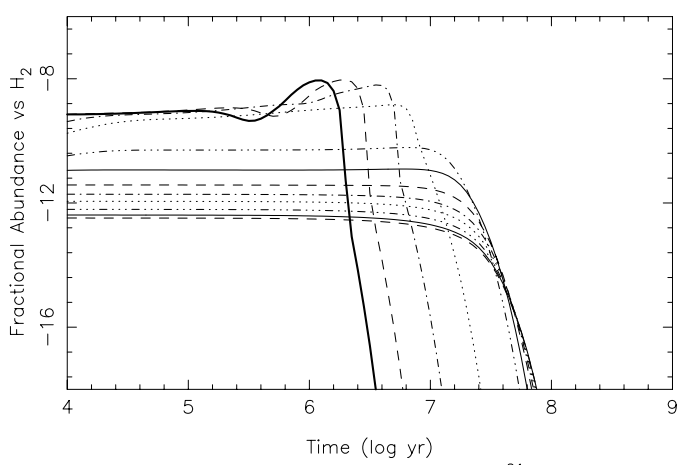

$\mathrm{H} 2 \mathrm{CO}$ abundance $\mathrm{S}=1 \mathrm{k}=1 \mathrm{O}^{21}$

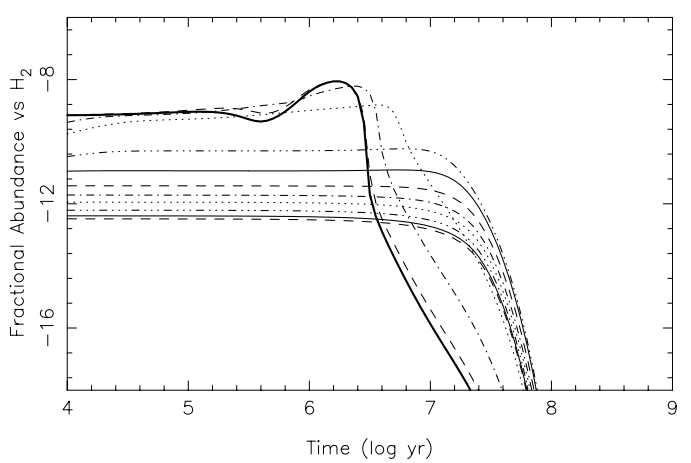

$\mathrm{H} 2 \mathrm{CO}$ abundance $\mathrm{S}=1 \mathrm{k}=10^{22}$

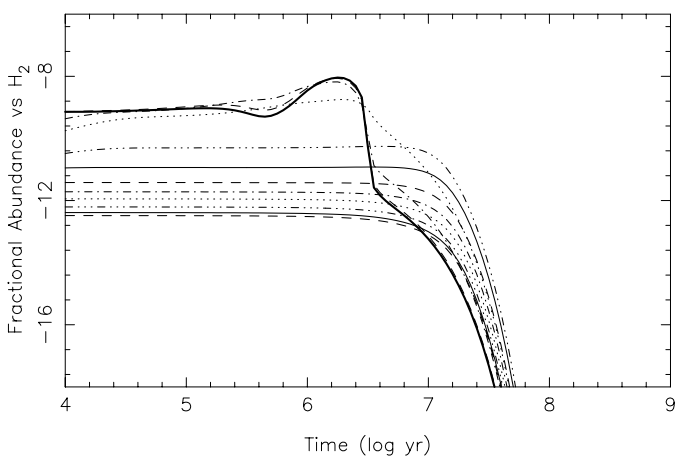

H2CO abundance $\mathrm{S}=1 \quad \mathrm{k}=10^{23}$

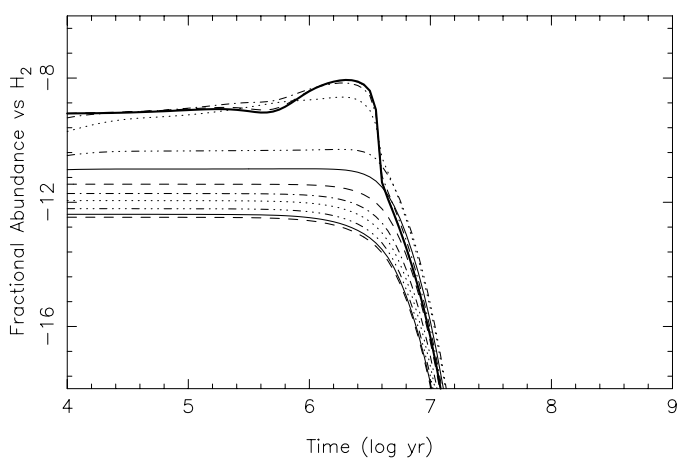

Fig. 5. $\mathrm{H}_{2} \mathrm{CO}$ abundances. Grain model A.

of up to 10 orders of magnitude in $\mathrm{CH}_{3} \mathrm{OH}$ abundance between the centre and the edge of the cloud. The abundance of $\mathrm{C}_{2} \mathrm{H}$ however, is much more constant with respect to depth into the cloud. Until freeze-out occurs, $\mathrm{C}_{2} \mathrm{H}$ has a range of about 2 orders of magnitude between the centre and edge of the cloud, peaking in the shell with depth $=1.71 \times 10^{18} \mathrm{~cm}$,
$\mathrm{CN}$ abundance $\mathrm{S}=1 \mathrm{k}=0$

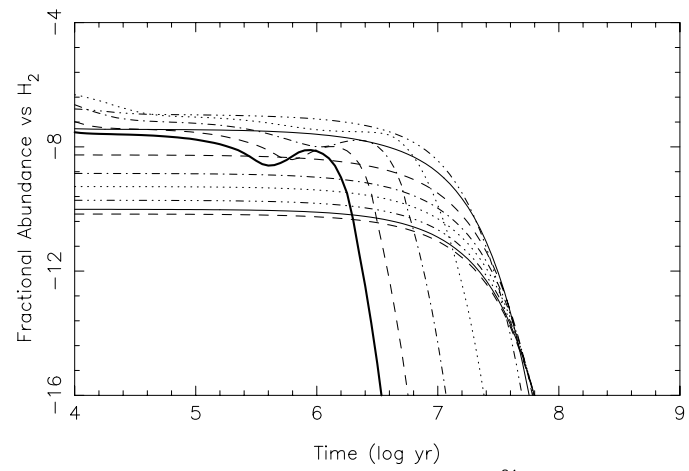

$\mathrm{CN}$ abundance $\mathrm{S}=1 \quad k=10^{21}$

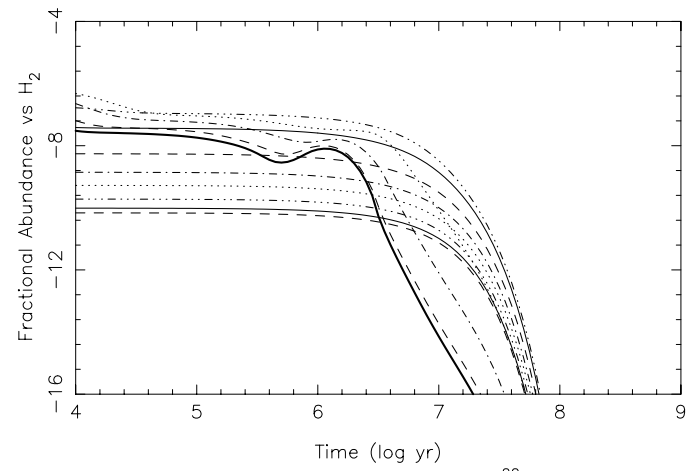

$\mathrm{CN}$ abundance $\mathrm{S}=1 \mathrm{k}=10^{22}$

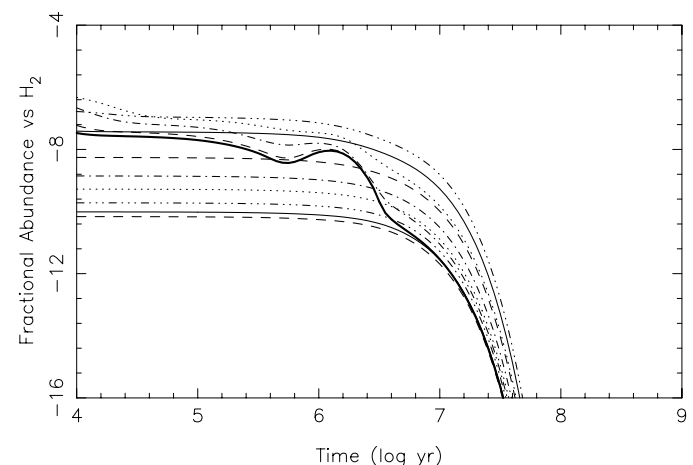

CN obundance $S=1 \quad k=10^{23}$

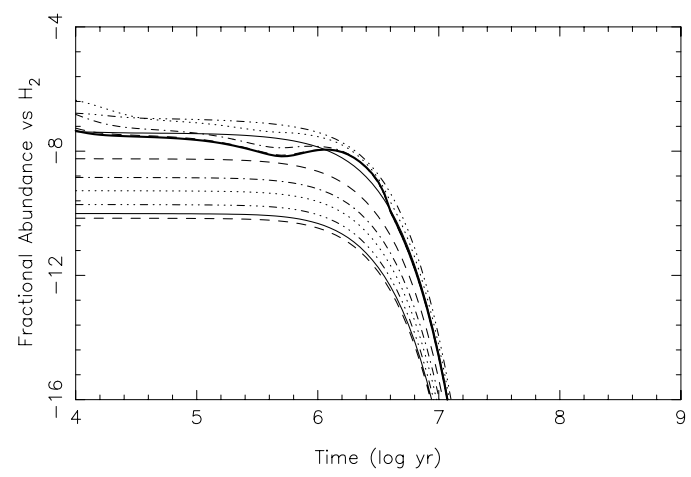

Fig. 6. $\mathrm{CN}$ abundances. Grain model A.

$A_{V}=2.0 \mathrm{mag}, n\left(\mathrm{H}_{2}\right)=1.76 \times 10^{3} \mathrm{~cm}^{-3}$. Without diffusion, these abundances persist until about $10^{5.8}$ years, when the $\mathrm{C}_{2} \mathrm{H}$ abundance at the centre begins to fall rapidly due to adsorption onto grains. However, with increasing $K$, the $\mathrm{C}_{2} \mathrm{H}$ abundance at the cloud centre remains at over $10^{-9}$ for longer, peaking in abundance at around a million years. The peak abundance also 
$\mathrm{HCN}$ abundance $S=1 \mathrm{k}=0$

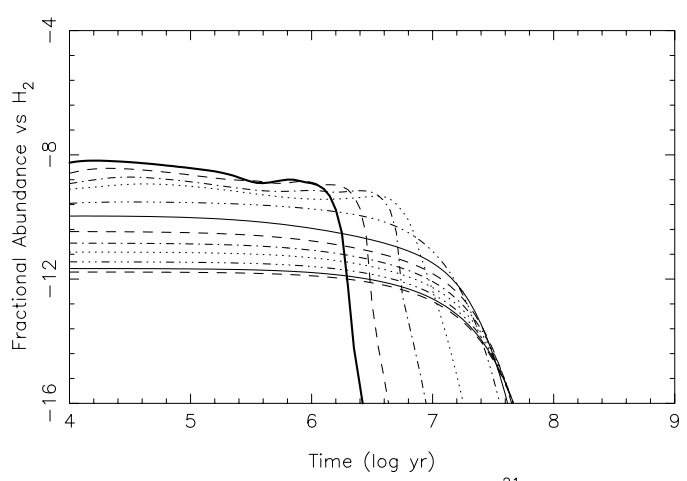

$\mathrm{HCN}$ abundance $\mathrm{S}=1 \mathrm{k}=10^{21}$

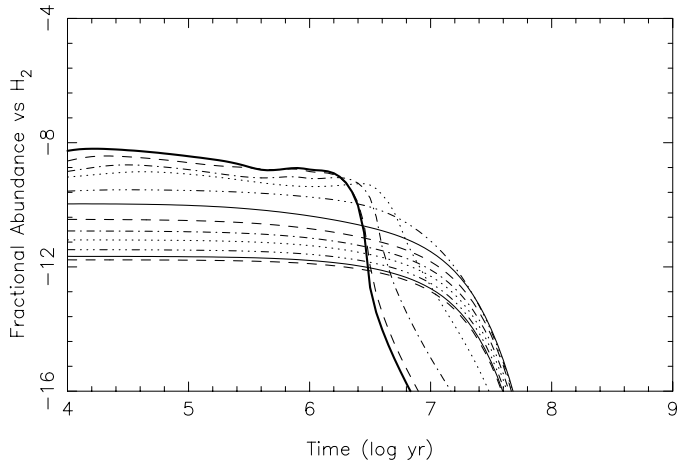

$\mathrm{HCN}$ abundance $\mathrm{S}=1 \mathrm{k}=10^{22}$

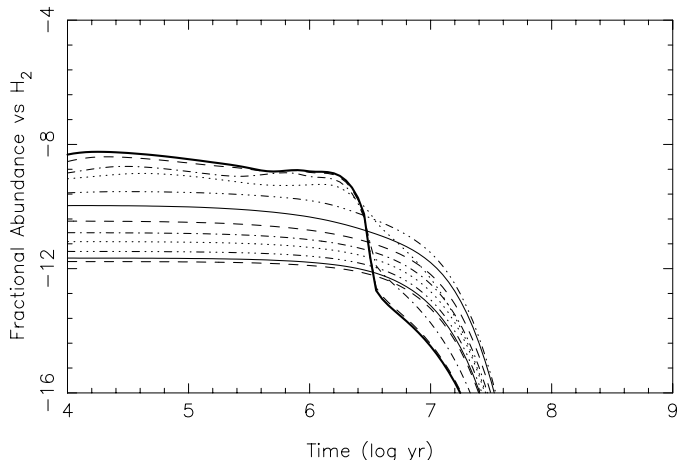

HCN abundance $S=1 \quad k=10^{23}$

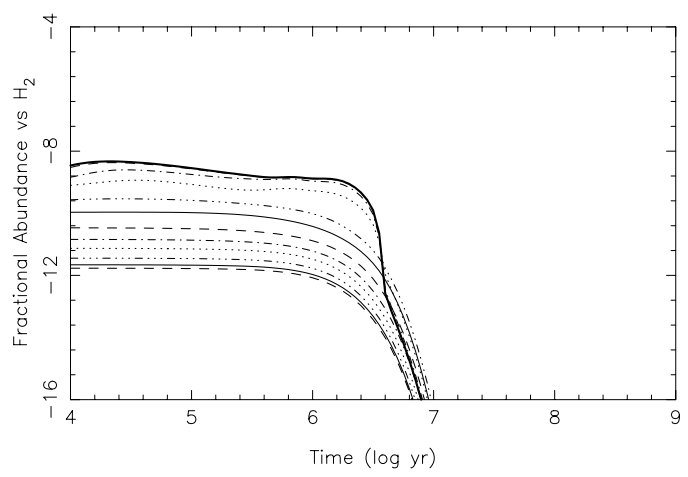

Fig. 7. HCN abundances. Grain model A.

increases with increasing $K$. As is the case with $\mathrm{CH}_{3} \mathrm{OH}$, diffusion can increase the abundance of $\mathrm{C}_{2} \mathrm{H}$ at the centre of the cloud by many orders of magnitude after $10^{6}$ years.

$\mathrm{H}$ does not freeze out, but the plots in Fig. 9 show the dependence of $\mathrm{H}$ on diffusion coefficient. The fractional abundance of $\mathrm{H}$ at the centre increases very slightly as $K$ increases.
$\mathrm{HCO}+$ abundance $\mathrm{S}=1 \mathrm{k}=0$

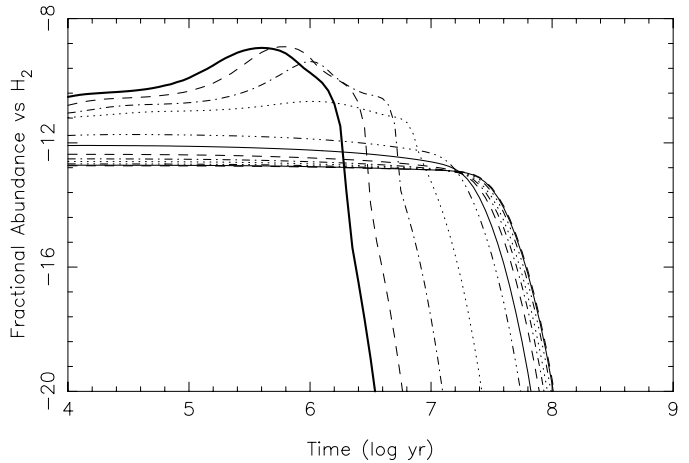

$\mathrm{HCO}+$ abundance $\mathrm{S}=1 \quad k=10^{21}$

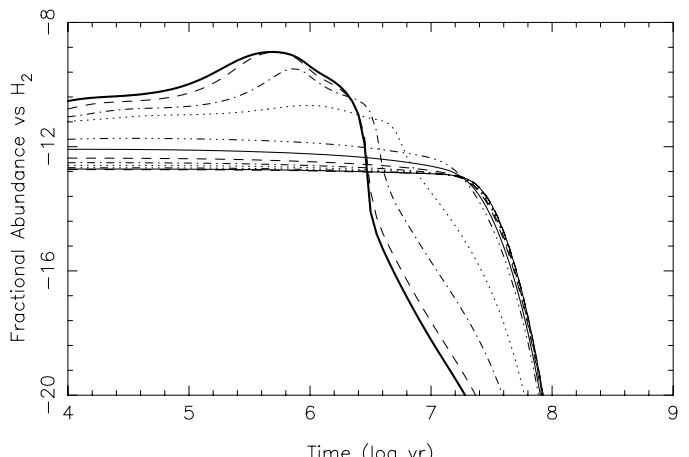

$\mathrm{HCO}+$ obundance $\mathrm{S}=1 \quad k=10^{22}$

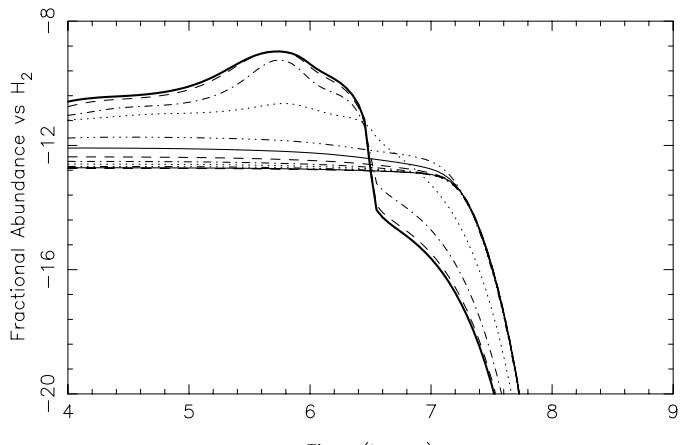

HCO+ abundance $\mathrm{S}=1 \quad k=10^{23}$

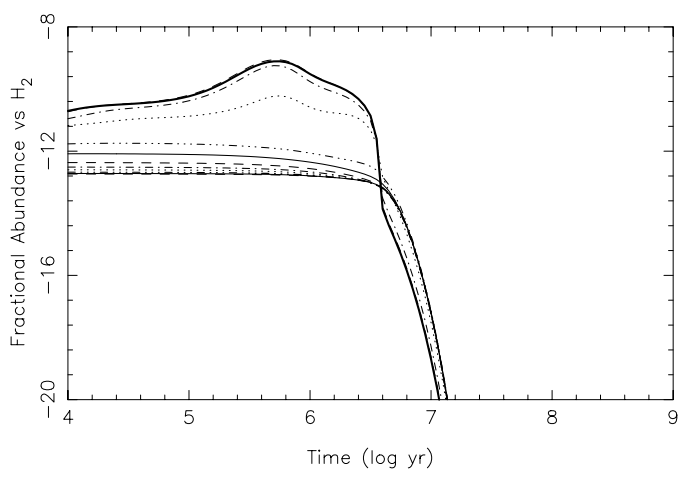

Fig. 8. $\mathrm{HCO}^{+}$abundances. Grain model A.

However, the abundance of hydrogen at the edge decreases considerably when $K=10^{23} \mathrm{~cm}^{2} \mathrm{~s}^{-1}$, down to an order of magnitude lower than the diffusionless model. The results for $\mathrm{H}$ are compared with the work of Willacy et al. (2002) in Sect. 5, below. 

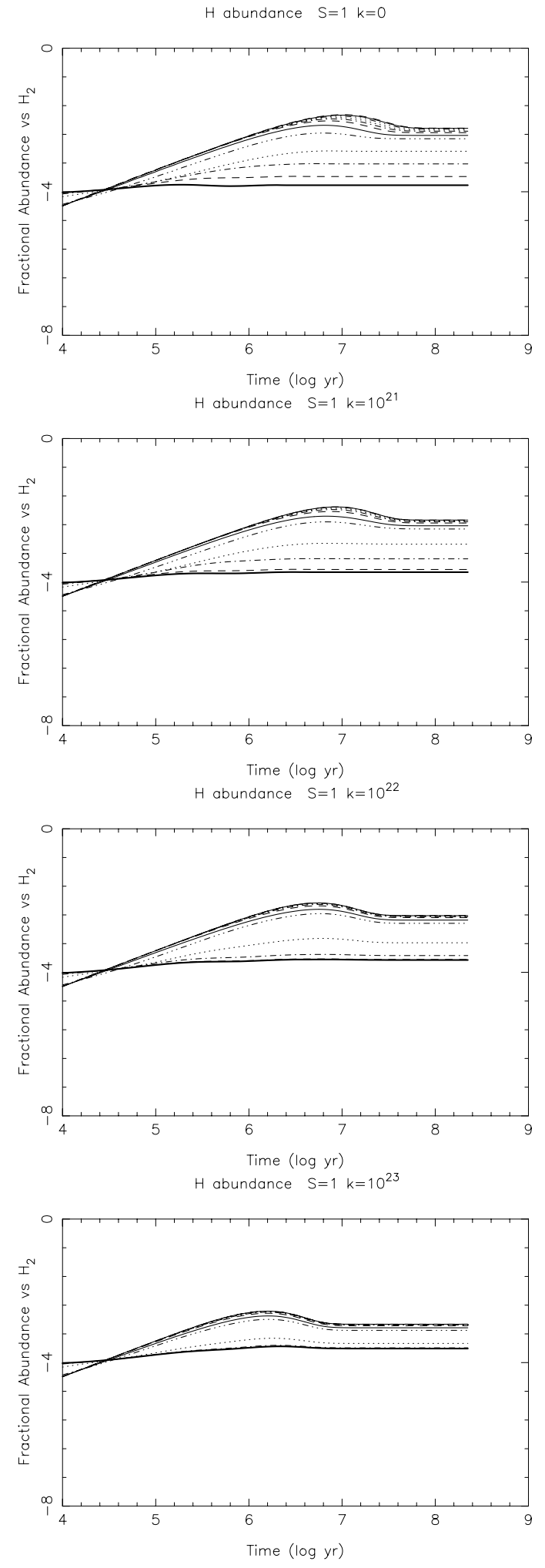

Fig. 9. $\mathrm{H}$ abundances. Grain model A.

\subsubsection{Disappearance time}

We will use the term "disappearance time" to describe the time at which a species is depleted from the gas and the abundance drops below a level such that it is unobservable. For clarity, it is useful to use the minimum abundance line (the time axis) on the plots to compare disappearance time (hereafter $\tau_{\text {dis }}$ ) between models, although it is not possible to observe species at that abundance.

There is only about an order of magnitude difference in $\tau_{\text {dis }}$ between species, for the models with $K=0 \mathrm{~cm}^{2} \mathrm{~s}^{-1}$. With $K=10^{21} \mathrm{~cm}^{2} \mathrm{~s}^{-1}$ we see a marked increase in $\tau_{\text {dis }}$ for most species. The effect is stronger for certain species. $\tau_{\text {dis }}$ for $\mathrm{NH}_{3}$ and $\mathrm{H}_{2} \mathrm{O}$ is increased by over an order of magnitude. In contrast $\mathrm{C}_{2} \mathrm{H}$ and $\mathrm{HCO}^{+}$are affected less strongly. This is an effect of the chemistry, as $\mathrm{NH}_{3}$ and $\mathrm{H}_{2} \mathrm{O}$ continue to form from chemical reactions late into the evolution of the cloud, delaying freezeout. As the diffusion coefficient is increased to $10^{22} \mathrm{~cm}^{2} \mathrm{~s}^{-1}$, $\tau_{\text {dis }}$ at the centre changes only slightly. The abundance gradients across the cloud are lower at late times, the effects beginning to occur at $t \simeq 10^{5.5}$ years, but most noticeable after $10^{6.5}$ years. This is a result of the mixing of material in the cloud.

With further increasing values of $K$, the cloud becomes more homogenised, and $\tau_{\text {dis }}$ throughout the cloud is decreased. For instance, compare the abundance of $\mathrm{NH}_{3}$ at $t \geq 10^{7}$ years, in the $K=10^{22}$ and $K=10^{23} \mathrm{~cm}^{2} \mathrm{~s}^{-1}$ graphs (in Fig. 3). As diffusion becomes stronger the accretion timescale for the central region of the cloud becomes dominant, and material at the edge diffuses inwards. The gas-phase lifetime of the cloud is reduced for very high values of the diffusion coefficient, but can be increased significantly for moderate values of $K$.

\section{2. $S=1 / 3-$ model $B$}

For models $\mathrm{B}$ and $\mathrm{C}$ we show plots for $\mathrm{C}_{2} \mathrm{H}, \mathrm{CH}_{3} \mathrm{OH}$ and $\mathrm{NH}_{3}$ only. These species show some of the most important trends in molecular abundances with respect to different values of $K$ and $S$.

Figures 10, 11 and 12 show the abundances of molecules when the sticking coefficient is set to $S=1 / 3$ for each species. The most obvious effect is the lengthening of the gas phase lifetime of the cloud. The model calculation was set to stop at $3 \times 10^{8}$ years, which has caused the cut-off seen in the $\mathrm{NH}_{3}$ plots.

One interesting difference to the model A results is the Sshaped feature in the $\mathrm{C}_{2} \mathrm{H}$ plot. A similar shape is seen in the $\mathrm{C}_{2} \mathrm{H}, \mathrm{CN}$ and $\mathrm{H}_{2} \mathrm{CO}$ results for model $\mathrm{A}$, but is a much more marked effect in model B for all values of $K$. The S-shape is also seen in model $\mathrm{B}$ for $\mathrm{H}_{2} \mathrm{CO}$ and other hydrocarbon species not displayed in this paper. The $\mathrm{C}^{+}$and $\mathrm{O}$ atom abundances are important causes for the $\mathrm{S}$-shaped feature. $\mathrm{C}^{+}$is formed mainly from $\left[\mathrm{He}^{+}+\mathrm{CO}\right]$ or cosmic ray induced photoionisation of $\mathrm{C}$ atoms, depending on the relative abundances of $\mathrm{C}, \mathrm{CO}$ and $\mathrm{He}^{+}$at a given time. The main production route of $\mathrm{C}_{2} \mathrm{H}$ at the centre of the cloud is cosmic-ray induced photodissociation of $\mathrm{C}_{2} \mathrm{H}_{2}$, which is formed from $\mathrm{C}_{2} \mathrm{H}_{3}^{+}$. There are various reactions which form $\mathrm{C}_{2} \mathrm{H}_{3}^{+}$at the centre of the cloud. One of the most important of these, although not always the fastest reaction is $\left[\mathrm{C}^{+}+\mathrm{CH}_{4}\right]$ as the reactants are not produced by destruction reactions of $\mathrm{C}_{2} \mathrm{H}_{3}^{+}$. Examining the reaction rates at 100000 years shows that all these reaction rates lie within an order of magnitude, and each is significant for $\mathrm{C}_{2} \mathrm{H}_{3}^{+}$formation. 
$\mathrm{CH} 3 \mathrm{OH}$ abundance $\mathrm{S}=0.333 \mathrm{k}=0$

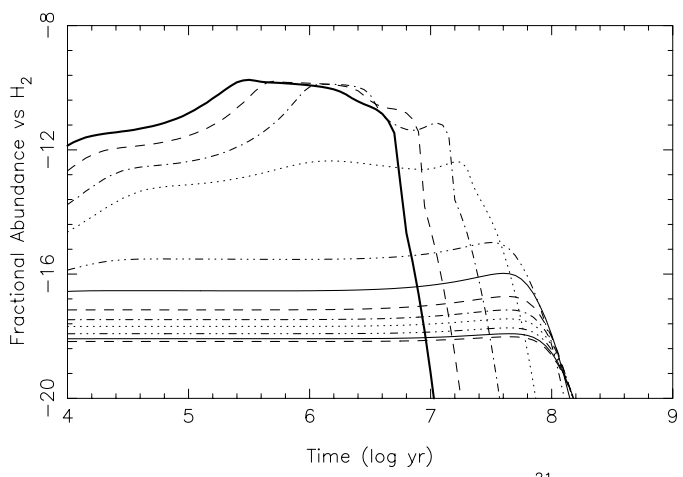

$\mathrm{CH} 3 \mathrm{OH}$ abundance $\mathrm{S}=0.333 \mathrm{k}=10^{21}$

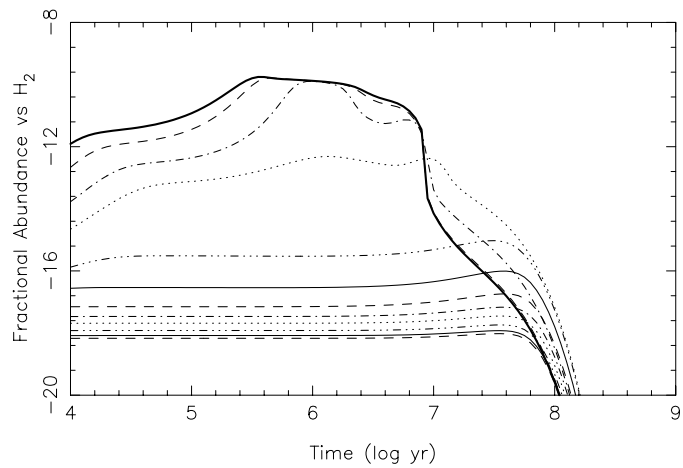

$\mathrm{CH} 3 \mathrm{OH}$ abundance $\mathrm{S}=0.333 \mathrm{k}=10^{22}$

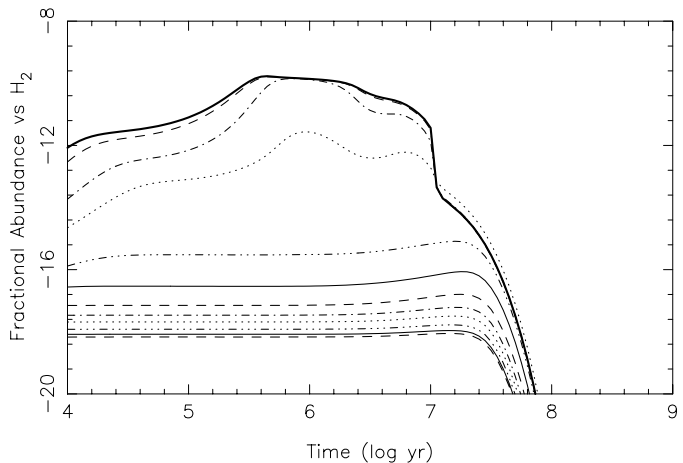

$\mathrm{CH} 3 \mathrm{OH}$ abundance $\mathrm{S}=0.333 \mathrm{k}=10^{23}$

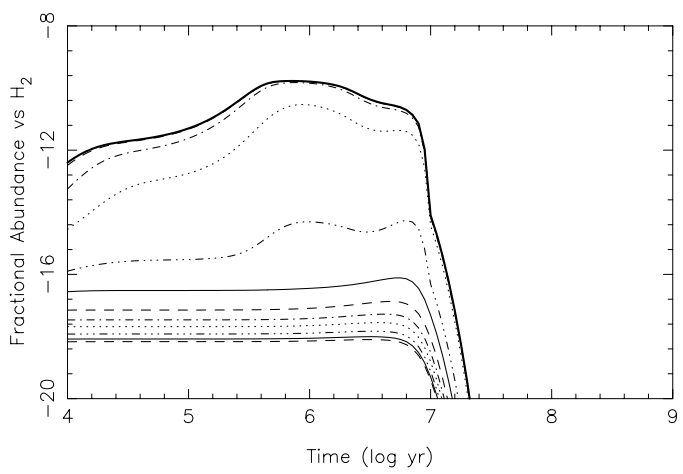

Fig. 10. $\mathrm{CH}_{3} \mathrm{OH}$ abundances, grain model $\mathrm{B}$. A heavy solid line marks the centre of cloud, each line plotted shows each alternate gridpoint.

$\mathrm{C}_{2} \mathrm{H}_{2}$ is formed from the electron recombination of $\mathrm{C}_{2} \mathrm{H}_{3}^{+}$, and cosmic ray induced photodissociation of $\mathrm{C}_{2} \mathrm{H}_{2}$ produces $\mathrm{C}_{2} \mathrm{H}$. The main destroyer of $\mathrm{C}_{2} \mathrm{H}$ at the cloud centre is $\mathrm{O}$ atoms which react to form $\mathrm{CO}$ and $\mathrm{CH}$. The destruction rate decreases as $\mathrm{O}$ is
$\mathrm{C} 2 \mathrm{H}$ abundance $\mathrm{S}=0.333 \mathrm{k}=0$

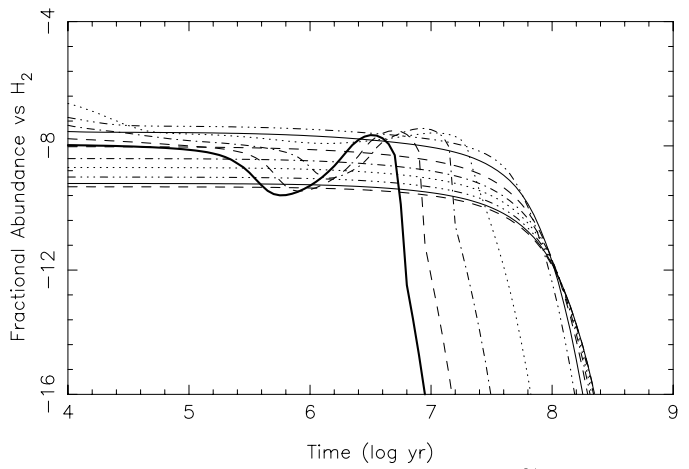

$\mathrm{C} 2 \mathrm{H}$ abundance $\mathrm{S}=0.333 \mathrm{k}=10^{21}$

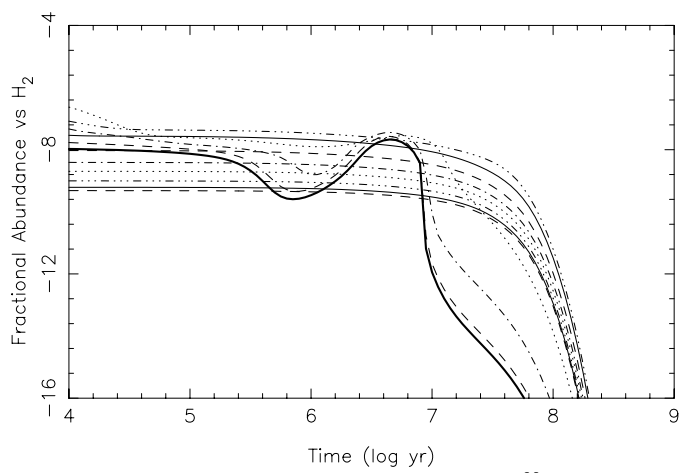

$\mathrm{C} 2 \mathrm{H}$ abundance $\mathrm{S}=0.333 \mathrm{k}=10^{22}$

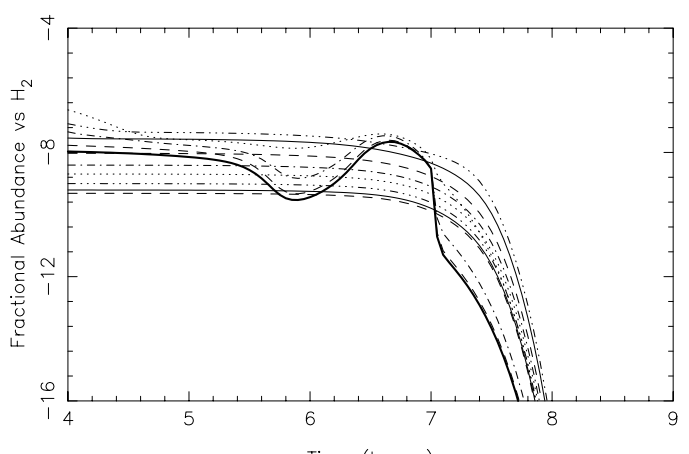

$\mathrm{C} 2 \mathrm{H}$ abundance $\mathrm{S}=0.333 \mathrm{k}=1 \mathrm{O}^{23}$

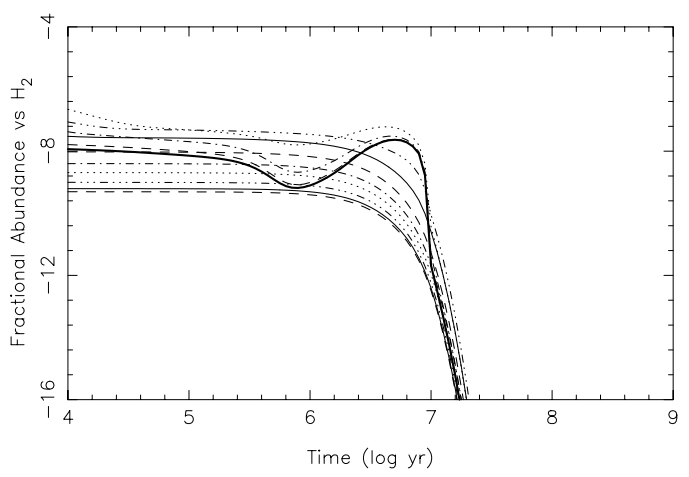

Fig. 11. $\mathrm{C}_{2} \mathrm{H}$ abundances. Grain model $\mathrm{B}$.

accreted onto dust grains. These two effects lead to an increase in the $\mathrm{C}_{2} \mathrm{H}$ abundance.

Apart from the reaction with $\mathrm{CH}_{4}$ which leads to $\mathrm{C}_{2} \mathrm{H}, \mathrm{C}^{+}$ is destroyed by reactions with $\mathrm{H}_{2} \mathrm{O}, \mathrm{H}_{2}$ and $\mathrm{O}_{2} . \mathrm{O}_{2}$ and $\mathrm{H}_{2} \mathrm{O}$ both peak at around $10^{6} \mathrm{yr}$ causing a drop in the $\mathrm{C}^{+}$abundance around this time. Thus, as a result of increasing $\mathrm{O}_{2}$ and $\mathrm{H}_{2} \mathrm{O}$, 
$\mathrm{NH3}$ abundance $\mathrm{S}=0.333 \mathrm{k}=0$

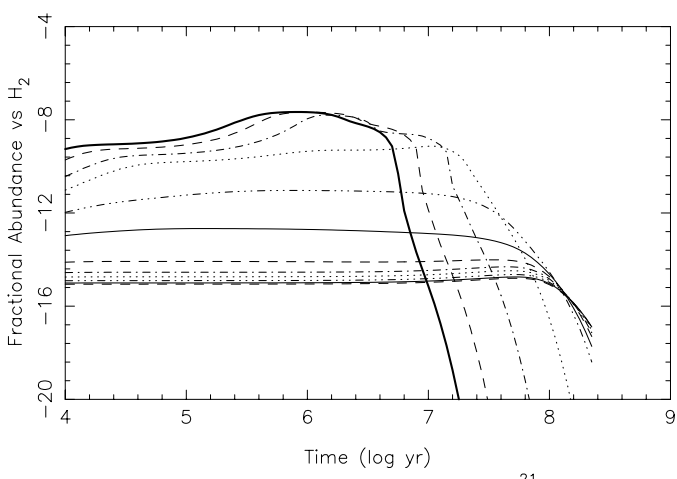

$\mathrm{NH} 3$ abundance $\mathrm{S}=0.333 \mathrm{k}=10^{21}$

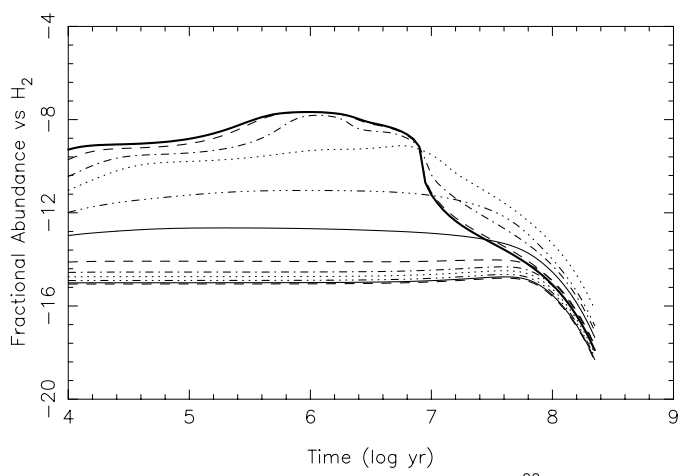

NH3 abundance $S=0.333 k=10^{22}$

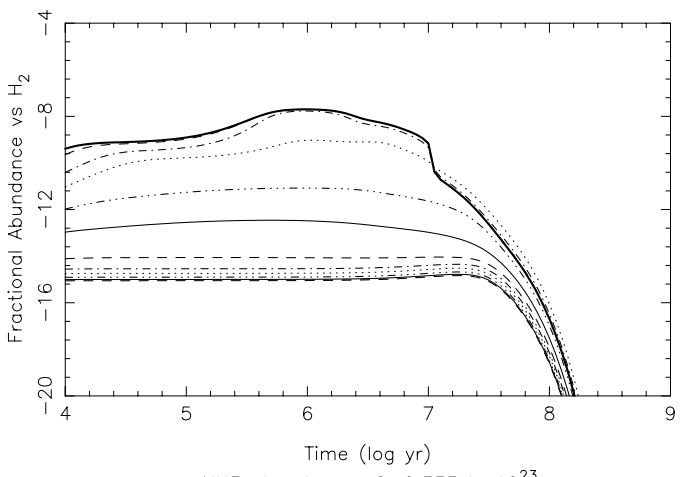

$\mathrm{NH} 3$ abundance $\mathrm{S}=0.333 \mathrm{k}=10^{23}$

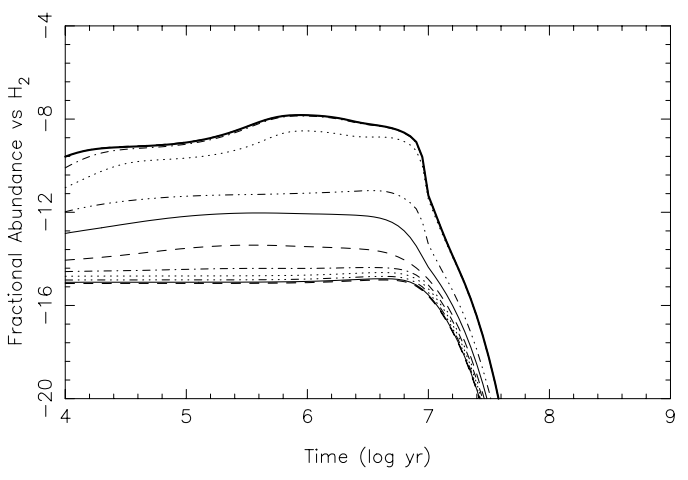

Fig. 12. $\mathrm{NH}_{3}$ abundances. Grain model B.

the $\mathrm{C}_{2} \mathrm{H}$ abundance also drops. Eventually, all $\mathrm{C}_{2} \mathrm{H}$ freezes out. In the $K=10^{21} \mathrm{~cm}^{2} \mathrm{~s}^{-1}$ and higher $K$ model results we can see that diffusion has a much stronger homogenising effect in model B, compared to model A. In other words, $\tau_{\text {dis }}$ varies less across the cloud. The longer disappearance times within the

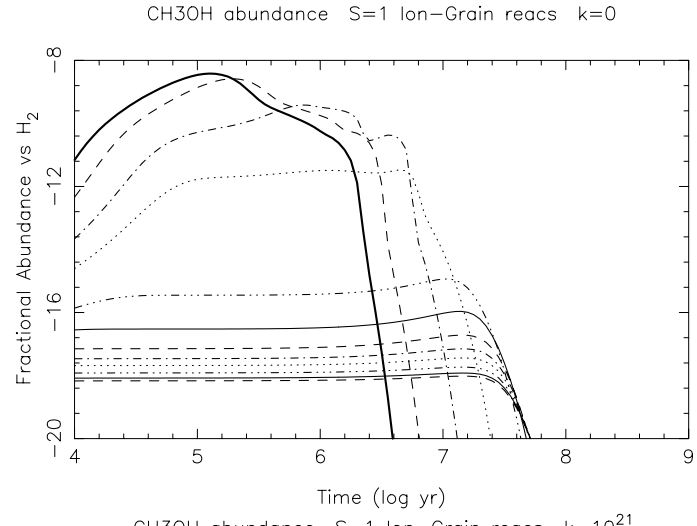

$\mathrm{CH} 3 \mathrm{OH}$ abundance $\mathrm{S}=1$ lon-Grain reacs $\mathrm{k}=10^{21}$

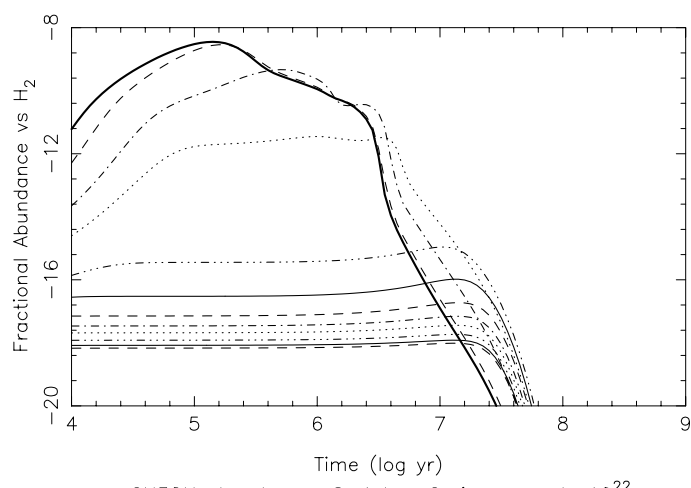

$\mathrm{CH} 3 \mathrm{OH}$ obundance $\mathrm{S}=1$ lon-Grain reacs $\mathrm{k}=10^{22}$

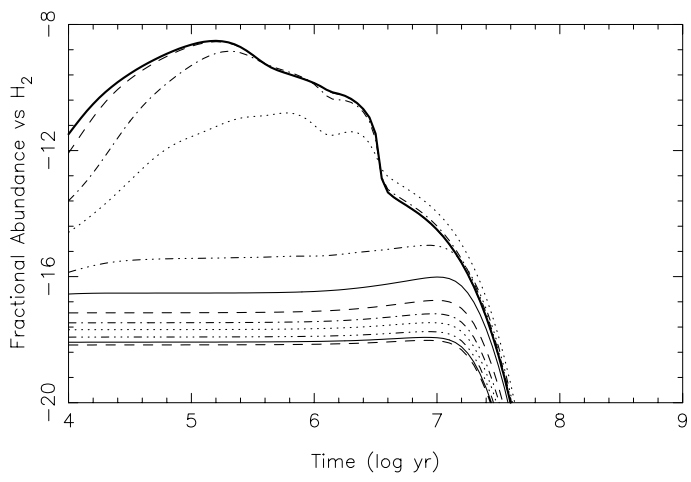

Fig. 13. $\mathrm{CH}_{3} \mathrm{OH}$ abundances, grain model $\mathrm{C}$. A heavy solid line marks the centre of cloud, each line plotted shows each alternate gridpoint.

cloud mean that the turbulent mixing has more time in which to modify abundances in the cloud.

\section{3. $S=1$, with ion/grain reactions - model $C$}

Model $\mathrm{C}$ has been calculated with values of $K$ up to $10^{22} \mathrm{~cm}^{2} \mathrm{~s}^{-1}$ as the $K=10^{23} \mathrm{~cm}^{2} \mathrm{~s}^{-1}$ models are very time consuming to run and may not represent realistic physical effects (see Sect. 4).

Figure 13 shows an increase in the peak abundance of methanol between models $\mathrm{A}$ and $\mathrm{C}$, although $\tau_{\text {dis }}$ is very similar between the two models. In model $\mathrm{C}, \mathrm{CH}_{3} \mathrm{OH}$ is about an order of magnitude more abundant at the peak, than the peak abundance in model A. Also the peak occurs at $10^{5.1}$ years for model $\mathrm{C}$ compared to $10^{5.2}$ years in model $\mathrm{A}$. There is 
$\mathrm{C} 2 \mathrm{H}$ abundance $\mathrm{S}=1$ lon-Grain reacs $\mathrm{k}=0$
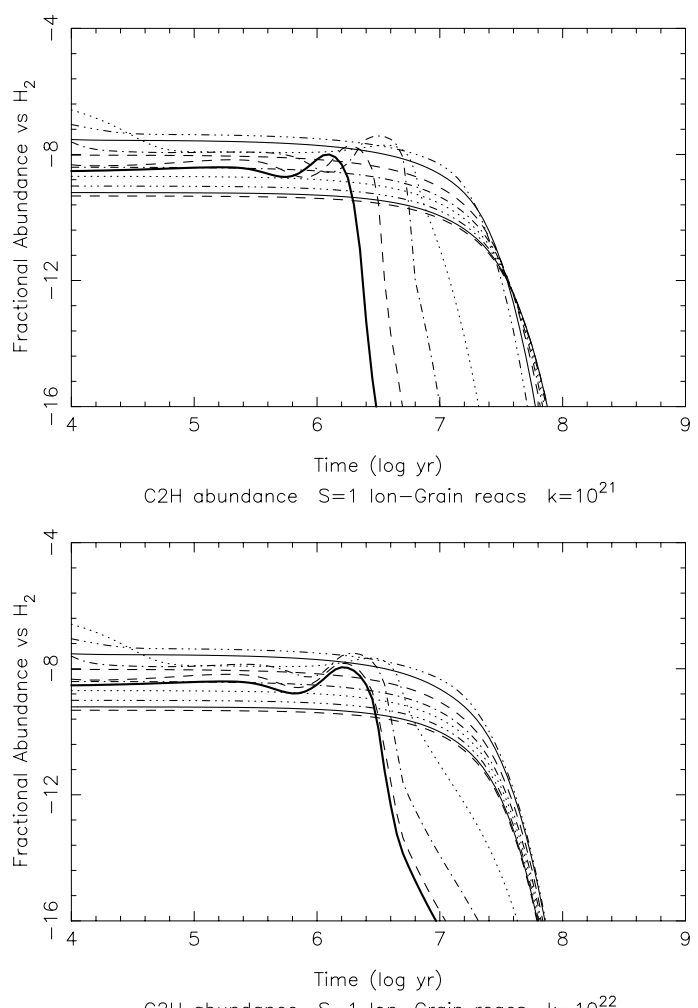

$\mathrm{C} 2 \mathrm{H}$ abundance $\mathrm{S}=1$ Ion-Grain reacs $\mathrm{k}=10^{22}$

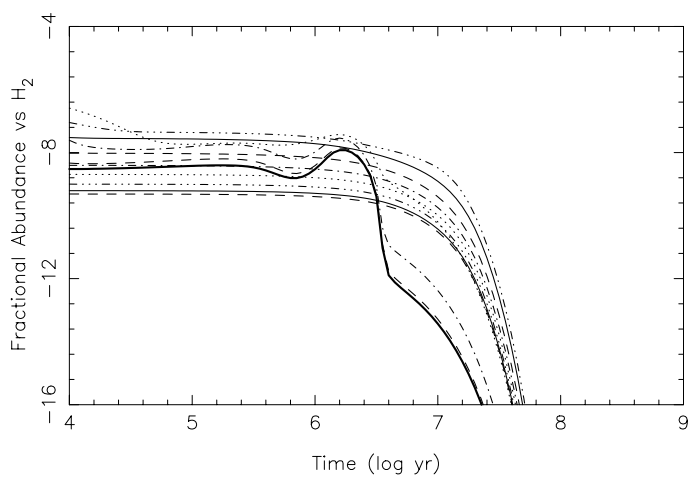

Fig. 14. $\mathrm{C}_{2} \mathrm{H}$ abundances. Grain model $\mathrm{C}$.

very little difference between the abundance of $\mathrm{C}_{2} \mathrm{H}$ in models A and C. The S-shaped feature is slightly less prominent in model $\mathrm{C}$. $\mathrm{NH}_{3}$ is slightly more abundant in model $\mathrm{C}$ up to its peak at $1 \mathrm{Myr}$, but the peak abundance is very similar to that in model A. For all these molecules $\tau_{\text {dis }}$ is almost exactly the same as for model A. The ion-grain reactions increase the abundance of certain molecules, as molecular ions hitting dust grains are recycled back into the gas phase as smaller neutral species which are the building blocks for larger species - such as $\mathrm{CH}_{3} \mathrm{OH}$. This explains why methanol has increased abundances before freeze-out occurs.

Model C shows a very similar response to $K$ as model A, although abundances of some molecules are higher before freeze-out. The sticking coefficient clearly has more influence on $\tau_{\text {dis }}$ than the ion-grain interactions we have considered here.
$\mathrm{NH3}$ abundance $\mathrm{S}=1$ lon-Grain reacs $k=0$

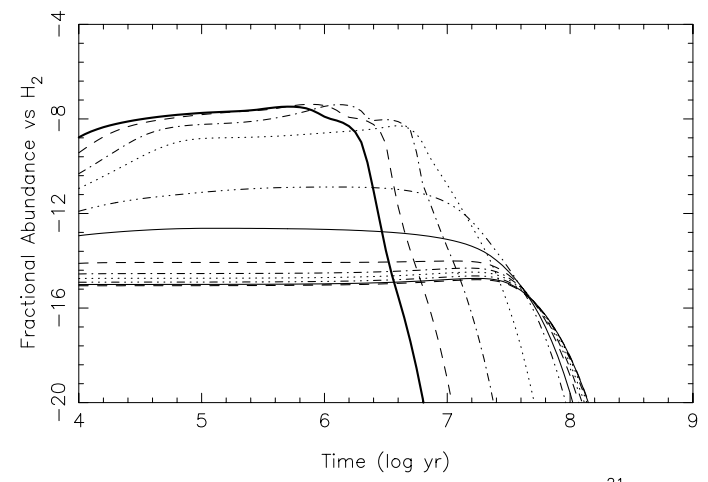

$\mathrm{NH3}$ abundance $\mathrm{S}=1$ lon-Grain reacs $k=10^{21}$

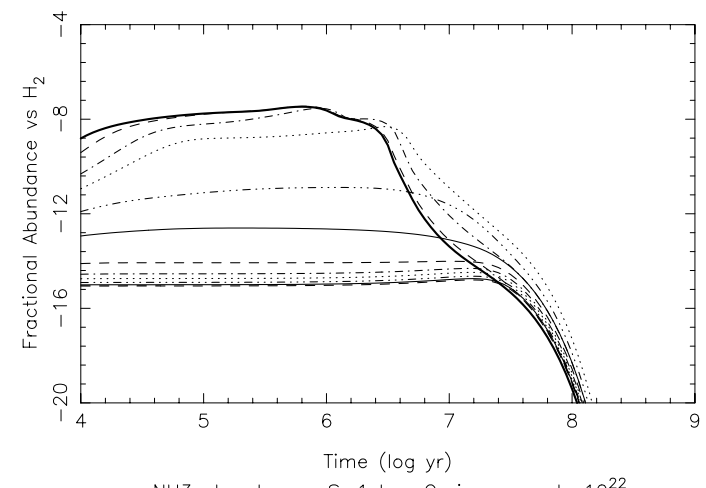

$\mathrm{NH} 3$ abundance $S=1$ lon-Grain reacs $k=10^{22}$

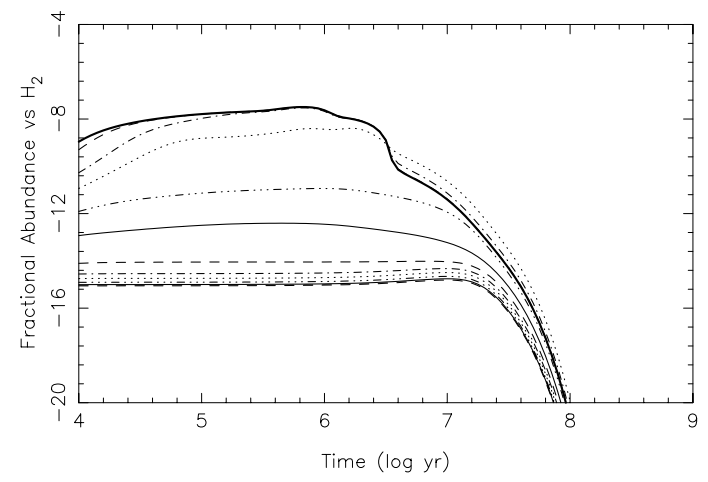

Fig. 15. $\mathrm{NH}_{3}$ abundances. Grain model $\mathrm{C}$.

\section{Discussion}

\subsection{Diffusion coefficients}

Larson (1981) and LKM82 compiled data from a number of clouds, in an attempt to find a relationship between line widths and cloud size. Larson first found correlation between cloud radius and velocity dispersion, and a similar relationship between velocity dispersion and the mass of a cloud. These are simple power law relations:

$\sigma=1.10 R(\mathrm{pc})^{0.38} \mathrm{~km} \mathrm{~s}^{-1}$,

$\sigma=0.42 M\left(M_{\odot}\right)^{0.2} \mathrm{~km} \mathrm{~s}^{-1}$.

Here $\sigma$ is defined as the total three-dimensional rms velocity of all internal motions within the cloud. This was revised by LKM82 for a sample of small dark clouds, as the work 
by Larson included many larger clouds and only a few small objects.

For objects with radii between 0.19 and 12 pc, LKM82 found

$v_{\mathrm{t}}=0.58 R(\mathrm{pc})^{0.48} \mathrm{~km} \mathrm{~s}^{-1}$,

$v_{\mathrm{t}}=0.21 M\left(M_{\odot}\right)^{0.21} \mathrm{~km} \mathrm{~s}^{-1}$

where $v_{\mathrm{t}}$ is the turbulent velocity, which was obtained by correcting the total linewidth observed for thermal motion, so is not quite the same value as Larson's $\sigma$. Both authors find a good correlation between line width and cloud mass. Larson also gives a rough value of the velocity dispersion due to thermal effects in a $10 \mathrm{~K}$ cloud, $\sigma_{\mathrm{s}}=0.32 \mathrm{~km} \mathrm{~s}^{-1}$.

Using $R=1 \mathrm{pc}$ in Eq. (10) gives $\sigma=1.1 \times 10^{5} \mathrm{~cm}^{2} \mathrm{~s}^{-1}$, which with a mixing length $L=0.5 \mathrm{pc}=1.5 \times 10^{18} \mathrm{~cm}$, gives $K=1.65 \times 10^{23} \mathrm{~cm}^{2} \mathrm{~s}^{-1}$. This represents an upper limit to the diffusion coefficient for a cloud of $1 \mathrm{pc}$ radius.

This invokes very large mixing lengths and velocities, which some authors have criticised (particularly Rawlings \& Hartquist 1997). In highly fragmented clouds the mixing lengths may be much smaller. There is evidence (e.g. Langer 1995) that the molecular cloud TMC-1 is fragmented on sizes down to around $0.03 \mathrm{pc}$, with line widths $\sigma \sim$ $0.1 \mathrm{~km} \mathrm{~s}^{-1}$, which give a minimum $K=3 \times 10^{20} \mathrm{~cm}^{2} \mathrm{~s}^{-1}$. With Larson's dispersion velocity calculations, which probably overestimate $v_{\mathrm{t}}$, we need a mixing length of $0.003 \mathrm{pc}\left(\sim 10^{16} \mathrm{~cm}\right)$ to give $K=10^{21} \mathrm{~cm}^{2} \mathrm{~s}^{-1}$.

\subsection{Timescales of diffusion and accretion}

The time-scale for altering the abundance of species $i$ is given by:

$\left[\frac{1}{n_{i}} \frac{\mathrm{d} n_{i}}{\mathrm{~d} t}\right]^{-1}$.

Thus, for example, the timescale for the accretion term in the ODEs (Eq. (1)) is:

$$
\begin{aligned}
t_{\mathrm{acc}} & =\left[\frac{1}{n_{i}} A_{i}\right]^{-1} \\
& =\left[\frac{1}{n_{i}} R_{i} n_{i} n_{\mathrm{g}}\right]^{-1} \\
& =\left[R_{i} n_{\mathrm{g}}\right]^{-1} \\
& =\left[S_{i} \sigma_{\mathrm{g}} n_{\mathrm{g}} \overline{v_{i}}\right]^{-1} .
\end{aligned}
$$

Similarly, for diffusion we can write down a diffusion timescale for species $i$ as:

$$
\begin{aligned}
t_{\text {diff }} & =\left[\frac{1}{n_{i}} \frac{\mathrm{d} \phi_{i}}{\mathrm{~d} z}\right]^{-1} \\
& =\left\{\frac{1}{n_{i}} \frac{\mathrm{d}}{\mathrm{d} z}\left[K n\left(\mathrm{H}_{2}\right) \frac{\mathrm{d} f_{i}}{\mathrm{~d} z}\right]\right\}^{-1} .
\end{aligned}
$$

From Eq. (14), it is clear that the time-scale depends on the abundance gradient between cells - a larger gradient gives a shorter diffusion time-scale. The timescale of diffusion for a given cell is difficult to calculate, as it is a complicated function with two differential terms. However, it is possible to give an upper limit for the transport timescale, $\tau_{\text {diffusion }} \leq R^{2} / K$ (Xie et al. 1995), based on the fact that the $v_{\mathrm{t}}$ term in $K$ is a random velocity. This timescale is much larger than typical chemical timescales, and yet turbulent diffusion greatly affects the chemistry of the cloud.

It is clear that turbulence affects chemistry on a much faster timescale, which is dependent on the density scale heights of both molecular hydrogen and the other species in the model across the grid.

The dependence of total density on accretion timescale causes a large abundance gradient clearly seen in Figs. 1-15. Since the accretion time-scale for any species is independent of its abundance, the longer disappearance times at the centre of the cloud with increased $K$ are caused by inward diffusion of material from the outer regions of the cloud. With high $K$, the adsorption timescale of the dense, central regions of the cloud starts to become dominant, as material from the edges diffuses inwards. This explains the decrease in overall $\tau_{\text {dis }}$ of the cloud for $K=10^{23} \mathrm{~cm}^{2} \mathrm{~s}^{-1}$.

\section{Conclusions}

The models of Xie et al. (1995) required diffusion coefficients $\geq 10^{23} \mathrm{~cm}^{2} \mathrm{~s}^{-1}$ in order to significantly alter the steady-state chemical abundances within their model cloud. One of the main criticisms made of the work of Xie et al., by Rawlings \& Hartquist (1997), was that Xie et al.'s values of $K$ were unphysically large.

Examining Eq. (3) and the values of turbulent velocity $v_{\mathrm{t}}$ found by LKM (1982) and Larson (1981), it is clear that to use a diffusion coefficient $K=10^{21} \mathrm{~cm}^{2} \mathrm{~s}^{-1}$ we can adopt values of the turbulent scale length $L$ as small as $0.01 \mathrm{pc}$. This is feasible in the context of a cloud core of radius $1 \mathrm{pc}$, and abundance variations on this length scale should be observable in nearby clouds.

We have shown that diffusion coefficients of $10^{21} \mathrm{~cm}^{2} \mathrm{~s}^{-1}$ can be sufficient to delay the freeze-out of gas phase species onto grains for several million years - increasing the time for which molecules are observable in gas at densities $\geq 10^{4} \mathrm{~cm}^{-3}$.

Decreasing the sticking coefficient from unity to $1 / 3$ can greatly affect species abundances, partly due to the increased timescales involved. Even at early times before depletion begins to dominate the chemistry, there can be large differences in abundances. Diffusion has a stronger effect with decreased sticking coefficient.

Willacy et al. (2002) have reported results from a similar model, which includes desorption from grains and grain surface reactions. They found that the $\mathrm{H}_{2} \mathrm{O}$ abundance decreases by about an order of magnitude at the centre of the cloud at $5 \mathrm{Myr}$, between their $K=10^{21}$ and $K=10^{23} \mathrm{~cm}^{2} \mathrm{~s}^{-1}$ models. In contrast, our results show an increase in abundance, which can be explained by the fact that Willacy et al. allowed the grains to mix throughout the cloud. Molecules which accreted onto grains at the centre can then be released from the grains at the edge of the cloud and return to the centre by diffusive processes. The central $\mathrm{C}^{+}$abundance increases with $K$ in 
Willacy et al.'s models due to this process, which causes the decrease in $\mathrm{H}_{2} \mathrm{O}$ abundance. We have assumed that the dust grains are static, and that no desorption processes occur.

There are also differences in the $\mathrm{H}$ abundance, which Willacy et al. found increases by up to 3 orders of magnitude at the cloud centre with increasing $K$. We find only a very small increase in the central $\mathrm{H}$ abundance with $K$, although at the cloud edge we find a marked decrease in the $\mathrm{H}$ abundance with increasing $K$. The column densities from our models (not shown here) reflect the abundances at the edge of the cloud, as the abundance there is significantly larger than at the centre and thus dominates the column density. Therefore, in contrast to Willacy et al., in our models the overall H column density decreases as $K$ increases. Willacy et al. considered $\mathrm{H}_{2}$ photodissociation and self-shielding, which explains the differences, as the extra $\mathrm{H}$ produced by photodissociation would be expected to significantly increase the abundances at the centre of the cloud when the diffusion coefficient is large.

The model results are difficult to compare with observation in any detailed manner but there is general agreement to within an order of magnitude between the abundances observed at the TMC-1 $(\mathrm{CP})$ position for $\mathrm{C}_{2} \mathrm{H}, \mathrm{NH}_{3}, \mathrm{HCN}$ and $\mathrm{HCO}^{+}$at around $10^{6}$ years for $K=10^{-21} \mathrm{~cm}^{2} \mathrm{~s}^{-1}$ in model A. However, both $\mathrm{CH}_{3} \mathrm{OH}$ and $\mathrm{H}_{2} \mathrm{CO}$ are underproduced by around an order of magnitude or so. Turner (1998) has shown that, in translucent clouds, the observed methanol abundance is some four orders of magnitude larger than predicted by gas-phase models, thus arguing for an active surface chemistry in these objects. In our models we get a much improved agreement between observed and predicted $\mathrm{CH}_{3} \mathrm{OH}$ and $\mathrm{H}_{2} \mathrm{CO}$ abundances in Model C. Note that these enhanced gas-phase abundances are not the result of an active surface chemistry but rather due to ion-grain collisions which return neutral products directly to the gas phase with an abundance larger than that in Model A. Thus at $10^{6}$ years, the water abundance in Model C is about 10 times that in Model A. In addition, the abundance of $\mathrm{C}^{+}$is reduced by about an order of magnitude in Model $\mathrm{C}$ since grain-assisted recombination is faster than other loss routes. Since $\mathrm{C}^{+}$is an important loss route for gas-phase methanol, these two effects - increased formation via an increased water abundance, and decreased loss through a reduction in the $\mathrm{C}^{+}$abundance - result in a substantially increased methanol abundance in Model C.

Diffusion models without accretion affect species with a natural depth dependent abundance gradient most strongly, e.g. $\mathrm{CH}_{3} \mathrm{OH}$ or $\mathrm{H}_{2} \mathrm{O}$. Our results show that with the inclusion of grain accretion many other species, such as $\mathrm{C}_{2} \mathrm{H}$, whose abundances have less dependence on are also sigificantly affected by diffusion.
Very dense cores may have densities well over $10^{5} \mathrm{H}_{2} \mathrm{~cm}^{-3}$. For those cores the accretion times involved will create even higher radial abundance gradients than seen in these models. In addition to high densities, cores which contain protostars also have high velocity dispersions, which drives the diffusion. Our results suggest that diffusion should be even more important in modifying the chemistry in these objects than in quiescent cores.

Acknowledgements. We are grateful to the referee, Dr. B. E. Turner, for a constructive report on this manuscript. CJY acknowledges receipt of a PPARC studentship. Astrophysics at UMIST is supported by PPARC.

\section{References}

Bergin, E. A., Langer, W. D., \& Goldsmith, P. F. 1995, ApJ, 441, 222 Bergin, E. A., Melnick, G., Stauffer, J. R., et al. 2000, ApJ, 539, L129 Chièze, J.-P. 1987, A\&A, 171, 225

Chièze, J.-P., \& Pineau des Forêts, G. 1987, A\&A, 183, 98

Chièze, J.-P., \& Pineau des Forêts, G. 1989, A\&A, 221, 89

Chièze, J.-P., Pineau des Forêts, G., \& Herbst, E. 1991, ApJ, 373, 110

d'Hendecourt, L. B., Allamandola, L. J., \& Greenberg, J. M. 1985, A\&A, 152, 130

Dickens, J. E., Irvine, W. M., Snell, R. M., et al. 2000, ApJ, 542, 870

Farquhar, P. R., Millar, T. J., \& Herbst, E. 1994, MNRAS, 269, 641

Goldsmith, P. F., Melnick, G. J., Bergin, E. A., et al. 2000, ApJ, 539, L123

Kleiner, S. C., \& Dickman, R. L. 1987, ApJ, 312, 837

Hindmarsh, A. C., \& Cohen, S. D. 1994, Numerical Mathematics Group, LLNL

Kitchin, C. R. 1987, in Stars, Nebulae and the Interstellar Medium, (Adam Hilger), 119

Larson, R. B. 1981, MNRAS, 194, 809

Langer, W. D., Velusamy, T., Kuiper, T. B. H., et al. 1995, ApJ, 453, 293

Le Teuff, Y. H., Millar, T. J., \& Markwick, A. J. 2000, A\&AS, 146, 157

Leung, C. M., Kutner, M. L., \& Mead, K. N. 1982, ApJ, 262, 583

Leung, C. M., Herbst, E., \& Huebner, W. F. 1984, ApJS, 56, 231

Markwick, A. J. 2000, Ph.D. Thesis (UMIST)

Myers, P. C. 1983, ApJ, 270, 105

Pratap, P., Dickens, J. E., Snell, R. L., et al. 1997, ApJ, 486, 862

Pineau des Forêts, G., Flower, D. R., \& Chièze, J.-P. 1992, MNRAS, 256, 247

Rawlings, J., \& Hartquist, T. 1997, ApJ, 487, 672

Scalo, J. M. 1984, ApJ, 277, 556

Scalo, J. M. 1987, in Interstellar Processes, ed. D. J. Hollenbach, \& H. A. Thronson (Dordrecht: D. Reidel), 349

Turner, B. E. 1998, ApJ, 501, 731

Willacy, K., Langer, W. D., \& Allen, M. 2002, ApJ, 573, 119

Xie, T., Allen, M., \& Langer, W. D. 1995, ApJ, 440, 674 